\title{
The Fusarium graminearum t-SNARE Sso2 Is Involved in Growth, Defense, and DON Accumulation and Virulence
}

\author{
Sean P. O’Mara, ${ }^{1}$ Karen Broz, ${ }^{2}$ Marike Boenisch, ${ }^{3}$ Zixuan Zhong, ${ }^{4}$ Yanhong Dong, ${ }^{3}$ and \\ H. Corby Kistler $2,3, \dagger$ \\ ${ }^{1}$ Department of Plant and Microbial Biology, University of Minnesota, St. Paul, MN 55108, U.S.A. \\ ${ }^{2}$ USDA ARS Cereal Disease Laboratory, St. Paul, MN 55108, U.S.A. \\ ${ }^{3}$ Department of Plant Pathology, University of Minnesota, St. Paul, MN 55108, U.S.A. \\ ${ }^{4}$ Research Center of Bioenergy and Bioremediation, College of Resources and Environment, Southwest University, Chongqing \\ 400715, P. R. China \\ Accepted 15 April 2020.
}

\begin{abstract}
The plant-pathogenic fungus Fusarium graminearum, causal agent of Fusarium head blight (FHB) disease on small grain cereals, produces toxic trichothecenes that require facilitated export for full virulence. Two potential modes of mycotoxin transport are membrane-bound transporters, which move toxins across cellular membranes, and $N$-ethylmaleimidesensitive factor attachment receptor (SNARE)-mediated vesicular transport, by which toxins may be packaged as cargo in vesicles bound for organelles or the plasma membrane. In this study, we show that deletion of a gene (Sso2) for a subapically localized t-SNARE protein results in growth alteration, increased sensitivity to xenobiotics, altered gene expression profiles, and reduced deoxynivalenol (DON) accumulation in vitro and in planta as well as reduced FHB symptoms on wheat. A double deletion mutant generated by crossing the $\Delta s s o 2$ deletion mutant with an ATP-binding cassette transporter deletion mutant $(\Delta a b c 1)$ resulted in an additive reduction in DON accumulation and almost complete loss of FHB symptoms in planta. These results suggest an important role of Sso2mediated subapical exocytosis in FHB progression and xenobiotic defense and are the first report of an additive reduction in F. graminearum DON accumulation upon deletion of two
\end{abstract}

${ }^{\dagger}$ Corresponding author: H. C. Kistler; hckist@umn.edu

RNA-seq data is available from GenBank under accession number PRJNA580269.

Mention of trade names or commercial products in this article solely for the purpose of providing specific information and does not imply recommendation or endorsement by the United States Department of Agriculture (USDA). USDA is an equal opportunity provider and employer.

Funding: Funding was provided by United States Department of Agriculture, United States Wheat and Barley Scab Initiative (award FY18-KI021) and from the Agriculture and Food Research Initiative of the National Institute of Food and Agriculture, United States Department of Agriculture (award 2018-67013-28512).

*The $\boldsymbol{e}$-Xtra logo stands for "electronic extra" and indicates that supplementary materials and a supplementary dataset are published online.

The author(s) declare no conflict of interest.

This article is in the public domain and not copyrightable. It may be freely reprinted with customary crediting of the source. The American Phytopathological Society, 2020. distinct modes of cellular export. This research provides useful information which may aid in formulating novel management plans of FHB or other destructive plant diseases.

Keywords: fungus-plant interactions, Fusarium head blight, mycotoxin, phytotoxin, secretion, transporters

The genus Fusarium contains many soil-borne and endophytic fungi as well as a number of economically important plant pathogens (Imazaki and Kadota 2015; Lofgren et al. 2018; Wachowska et al. 2017; Waweru et al. 2014). The species Fusarium graminearum has received considerable attention due to its role as a plant pathogen as well as its effects on animal and human health. F. graminearum is the causal agent of Fusarium head blight (FHB) disease of wheat and barley. In the United States, FHB outbreaks and management have resulted in annual losses in excess of $\$ 1.4$ billion (Wilson et al. 2018), and the disease remains a persistent problem. Current management techniques, such as crop rotation, variety resistance, fungicide use, and tillage have shown moderate success (Chen et al. 2019) but are unable to completely manage FHB.

F. graminearum produces numerous secondary metabolites, some of which act as virulence factors during infection and invasion of its hosts. The sesquiterpenoid trichothecenes are toxic secondary metabolites produced by $F$. graminearum (Chen et al. 2019). The trichothecene deoxynivalenol (DON) is essential for full virulence of $F$. graminearum on wheat (Proctor 1995) and may persist as a contaminant in affected grains. DON, like other trichothecenes, acts by binding to the peptidyltransferase domain of eukaryotic ribosomes, inhibiting protein synthesis (Fried and Warner 1981; Garreau de Loubresse et al. 2014). In addition, DON toxicosis in plants and animals can lead to DNA/RNA synthesis disruption, induction of apoptosis, ribotoxic stress, and membrane-directed cytotoxicity (Pestka 2007; Rocha et al. 2005). Thus DON, commonly referred to as a vomitoxin, is of special concern for human and livestock health.

While key enzymes required for DON biosynthesis are localized and enriched at the organized smooth endoplasmic reticulum (OSER) upon DON induction in $F$. graminearum (Boenisch et al. 2017, 2019), mechanisms by which DON is transported within toxigenic cells and exported are unknown. As DON is an effective protein synthesis inhibitor, $F$. graminearum presumably must be able to sequester, export, or otherwise avoid the activity of this toxin or risk self-inhibition. Within the core 
trichothecene biosynthetic gene cluster of $F$. graminearum is the gene Tri12 encoding a major facilitator superfamily transporter (FGSG_03541; FGRAMPH1_01G13119) (Proctor et al. 2009). Disruption of the Tri12 gene of $F$. sporotrichioides, a close relative of $F$. graminearum, results in highly reduced trichothecene accumulation in culture and reduced resistance to exogenously applied trichothecenes (Alexander et al. 1999). However, disruption of Tri12 in F. graminearum results in only slightly reduced DON accumulation (Menke et al. 2012). This suggests that, in $F$. graminearum, additional mechanisms for DON export may exist.

Other membrane-bound transporters, such as the ATP-binding cassette transporter Abc1 (FGSG_04580; FGRAMPH1_01G15627) may be involved in DON export. Abc1 has been shown to mediate transport of other secondary metabolites in $F$. graminearum. Deletion mutants of $A b c l$ show reduced virulence during wheat crown and root-rot infections, and expression of $A b c l$ is highest during the necrotrophic phase of infection (Gardiner et al. 2013). Strains of $F$. graminearum that produce DON or nivalenol (a C4-hydroxy derivative of DON) show reduced virulence on wheat, barley, and maize and altered mycotoxin production when $A b c l$ (referred to in this reference as $A b c 3$ ) is deleted (Abou Ammar et al. 2013). Abcl is also involved in transport of the $F$. graminearum estrogenic metabolite zearalenone and provides resistance to other antifungal compounds (Abou Ammar et al. 2013; Gardiner et al. 2013; Lee et al. 2011).

In addition to membrane-bound transporters, vesicular trafficking is an important mode of cellular transport. Vesicular transport plays a major role in signal transduction, vegetative growth, reproduction, secretion, effector recruitment, organelle movement, and vacuole formation (Hong et al. 2010; Hutagalung and Novick 2011; Zheng et al. 2015) and may also facilitate virulence and effector delivery in pathogenic fungi (Giraldo and Valent 2013). A localization study of green fluorescent protein (GFP)-tagged Tri12 indicated that the protein is found not only in the plasma membrane but also in highly motile vesicles that move between the site of DON synthesis (OSER) toward the vacuole and appear to fuse with the plasma membrane (Menke et al. 2012). A model has been proposed whereby Tri12-containing vesicles accumulate DON at the OSER and either sequester DON by fusion with the vacuole or traffic DON from OSER to outside the cell by fusion with the plasma membrane (Menke et al. 2012).

Vesicular transport is mediated by $N$-ethylmaleimidesensitive factor attachment receptor (SNARE) proteins. SNAREs can be classified as either v-SNAREs or t-SNAREs, based on their localization to vesicle and target membranes, respectively. Previous research has studied the role of SNAREs in growth, conidiation, sexual compatibility, and virulence of yeast and filamentous fungi (Hong et al. 2010; Kienle et al. 2009; Kuratsu et al. 2007; Li et al. 2019; Read 2011). The F. graminearum t-SNARE Sso2 (called GzSyn2 by Hong et al. [2010]) has been shown to localize to the subapical plasma membrane and septa but was not required for normal vegetative growth. Additionally, $\Delta$ sso 2 mutants were significantly reduced in virulence to barley (Hong et al. 2010). Since DON is not essential for full virulence of $F$. graminearum to barley (Maier et al. 2006) but is required for virulence to wheat (Proctor 1995), there is a need to explore the role of Sso2 in DON export and the virulence of $F$. graminearum to wheat. In this study, a deletion mutant of Sso2 (FGSG_09928; FGRAMPH1_01G06855) was generated and was analyzed for its sensitivity to xenobiotics and ability to accumulate DON and cause disease on wheat. For comparison and analysis of additive effects when eliminating two potential routes of toxin export, the $\Delta s s o 2$ mutant was crossed with an $\Delta a b c l$ deletion mutant.

\section{RESULTS}

\section{Confirmation of the $S s o 2$ deletion.}

The coding region of the $F$. graminearum Sso2 gene was deleted to assess its role in xenobiotic defense, DON accumulation, and in-planta virulence. Site-directed Sso2 deletion was performed by replacing the native gene with a gene for neomycin resistance (neoR). The mutant genotype was confirmed, using PCR, by selectively amplifying either the wild-type locus or its drug resistance-construct replacement (Supplementary Fig. S1). Five, independent, $\Delta$ sso2 transformation mutants were determined to have similar altered morphological phenotype, growth, and in-vitro deoxynivalenol accumulation (Supplementary Fig. S2), and one mutant ( $\Delta$ sso 2 5.1) was used for further analysis.

\section{Growth and phenotype of the $\Delta$ sso 2 deletion mutant.}

A confirmed $\Delta$ sso 2 mutant and an ectopic transformant were grown for 5 days on six different media routinely used for F. graminearum growth (Fig. 1). The colonies were assessed at 3 days for colony area, to determine growth differences, and at 5 days for coloration and other phenotypic differences. The $\Delta$ sso2 mutant showed coloration differences on half-strength potato dextrose agar (1/2 PDA), Czapek-Dox (CD), and

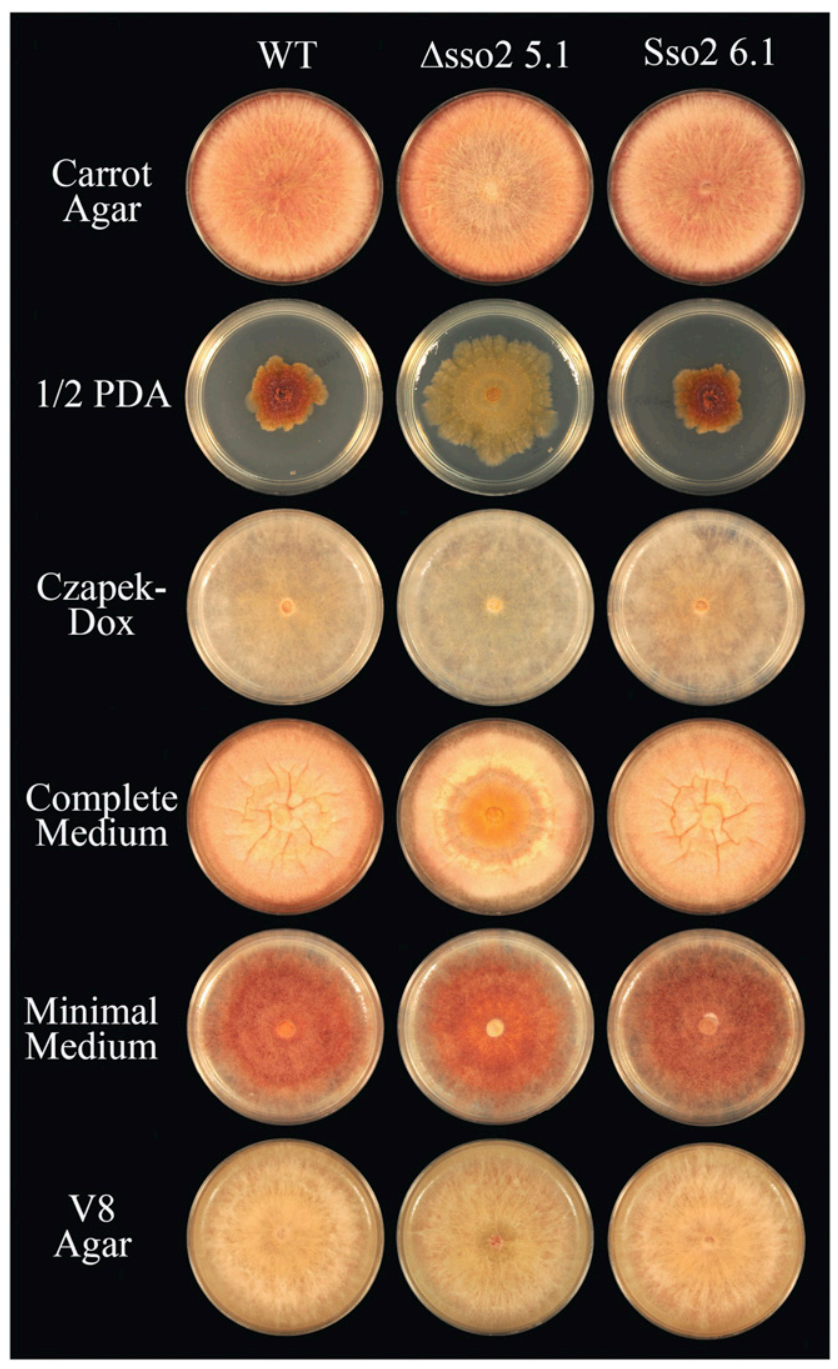

Fig. 1. Growth of Fusarium graminearum $\Delta$ sso2 mutant. Representative images of $F$. graminearum wild type and $\Delta s s o 2$ mutants grown on six kinds of media for 5 days. WT $=F$. graminearum $\mathrm{PH} 1, \Delta \mathrm{sso} 25.1=\Delta s s o 2$ mutant, Sso2 6.1 = ectopic transformant. 
complete medium (CM) (Fig. 1) and grew significantly faster on 1/2 PDA, CD, and minimal medium (MM) (Fig. 2). The ectopic transformant grew similarly to the wild type in all cases. To determine if the increased growth of the $\Delta$ sso 2 mutant was due to increased accumulation of biomass, wild-type $F$. graminearum and the $\Delta s s o 2$ mutant were grown in liquid half-strength potato dextrose broth (1/2 PDB) for 3 days. It was determined that the $\Delta s s o 2$ mutant does not accumulate significantly greater biomass than wild-type $F$. graminearum when grown in liquid $1 / 2 \mathrm{PDB}(t=1.83$, degree of freedom $[d f]=4$, $P=0.14)$. Also, upon closer examination, the $\Delta s s o 2$ mutant appears to form fewer branches than wild-type $F$. graminearum when grown on PDA medium (Fig. 3).

On $1 / 2$ PDA and CD, the $\Delta s s o 2$ mutant was observed to have different coloration from wild type (i.e., yellow rather than red) (Fig. 1), suggesting a potential role of Sso2 in the export of $F$. graminearum red pigments or the enzymes involved in their biosynthesis, a process that is $\mathrm{pH}$-sensitive. Additionally, when grown in $1 / 2$ PDB for 6 days, the $\Delta s s o 2$ mutant acidified the medium to a greater extent than did wild-type $F$. graminearum $(t=23.94$, $d f=2, P=0.002$ ), reaching a $\mathrm{pH}=4.78 \pm 0.01$ (mean \pm standard deviation) versus $\mathrm{pH}=5.99 \pm 0.09$, respectively. This indicates that $\mathrm{Sso} 2$ is involved in maintaining cellular $\mathrm{pH}$ homeostasis.

The $\Delta$ sso 2 mutant was grown on CM supplemented with xenobiotics for 5 days to assess its sensitivity to fungicides, plant defense compounds, and oxidative stress (Fig. 4A). The ectopic transformant was also included as a neomycin-resistance cassette control. There were no significant differences $(P>0.05)$ in the growth of either the $\Delta$ sso 2 mutant or ectopic transformant compared with that of wild-type $F$. graminearum on any of the xenobiotic supplemented cultures (Fig. 4A).

However, due to the increased growth of the $\Delta s s o 2$ mutant on $1 / 2$ PDA, the xenobiotic sensitivity test was repeated on this medium (Fig. 4B). The $\Delta$ sso 2 mutant grew faster than wildtype $F$. graminearum on the dimethyl sulfoxide (DMSO) carrier control, similar to growth on $1 / 2$ PDA alone (Fig. 2). For this reason, growth on 1/2 PDA supplemented with xenobiotics was analyzed as the percent growth compared with the DMSO control (Fig. 4C). The $\Delta$ sso 2 mutant had a greater reduction in growth compared with wild type on the fungicide tebuconazole but was equally reduced in growth on benomyl, indicating little or no change in effect of the benzimidazole fungicide. The $\Delta s s o 2$ mutant also had a significantly greater reduction in growth in the presence of the wheat phytoalexin 2-benzoxazolinone (BOA) and under oxidative stress by $\mathrm{H}_{2} \mathrm{O}_{2}$ (Fig. $4 \mathrm{C}$ ). BOA and $\mathrm{H}_{2} \mathrm{O}_{2}$ are of interest because $F$. graminearum would likely face these compounds during the induced response of wheat to infection (Desmond et al. 2008; Villagrasa et al. 2006). The differences in growth between the xenobiotic tests conducted on CM and $1 / 2$ PDA show that xenobiotic sensitivity is medium-specific, indicating that the importance of Sso2 to F. graminearum defense depends upon to its environmental context.

Because of the differential response of the mutant in xenobiotic tests, further analysis was conducted using Biolog phenotype microarray plates (Hayward, CA, U.S.A.). Wild-type $F$. graminearum and the $\Delta s s o 2$ mutant were grown on Biolog PM21-PM25 microarray plates for 3 days, screening for chemical sensitivity to 120 xenobiotics. The $\Delta s s o 2$ deletion showed increased sensitivity to many compounds tested, including numerous metal salts (e.g., magnesium chloride, sodium salicylate), antibiotic/antifungals (e.g., D-cycloserine, triclosan, hydrochloride, chloroquine), and phenothiazines (e.g., trifluoperazine, chloropromazine hydrochloride, thioridazine hydrochloride) (Supplementary Fig. S3). There were numerous compounds that showed little or no inhibitory activity to wild-type $F$. graminearum but did inhibit the $\Delta$ sso 2 mutant, including EDTA, compound 48/80, miltefosine, ammonium sulfate, cadmium chloride hydrate, dequalinium chloride, sodium cyanide, blastocidin hydrochloride, sodium benzoate, tobramycin, tetrazolium violet, and ibuprofen. These results indicate that Sso2-mediated endosomal membrane fusion plays an important role in tolerance to a broad range of xenobiotics and its function may not be replaced or replicated by other mechanisms of tolerance.

To analyze differences in carbon utilization of the $\Delta s s o 2$ mutant, wild-type and $\Delta$ sso $2 \mathrm{~F}$. graminearum were grown in Biolog SPF2 carbon utilization plates for 4 days. The $\Delta s s o 2$ mutant showed decreased growth on many mono- and disaccharide compounds, including maltose, turanose, D-cellobiose, D-fructose, D-mannitol, D-galactose, D-melibiose, gentiobiose, $\alpha$-methyl-D-galactoside, D-gluconic acid, D-sorbitol, and the $\beta$ glucoside salicin (Supplementary Fig. S4). These findings suggest that Sso2 may play an important role in endocytic uptake of sugars or perhaps the export of glycosidic linkage cleaving enzymes (e.g., glycoside hydrolases).

\section{Localization of a red fluorescent protein (RFP)-tagged Sso2.}

A Sso2-RFP-tagged strain was imaged after $30 \mathrm{~h}$ of growth in PDB to visualize the subcellular localization of Sso2. Sso2-RFP

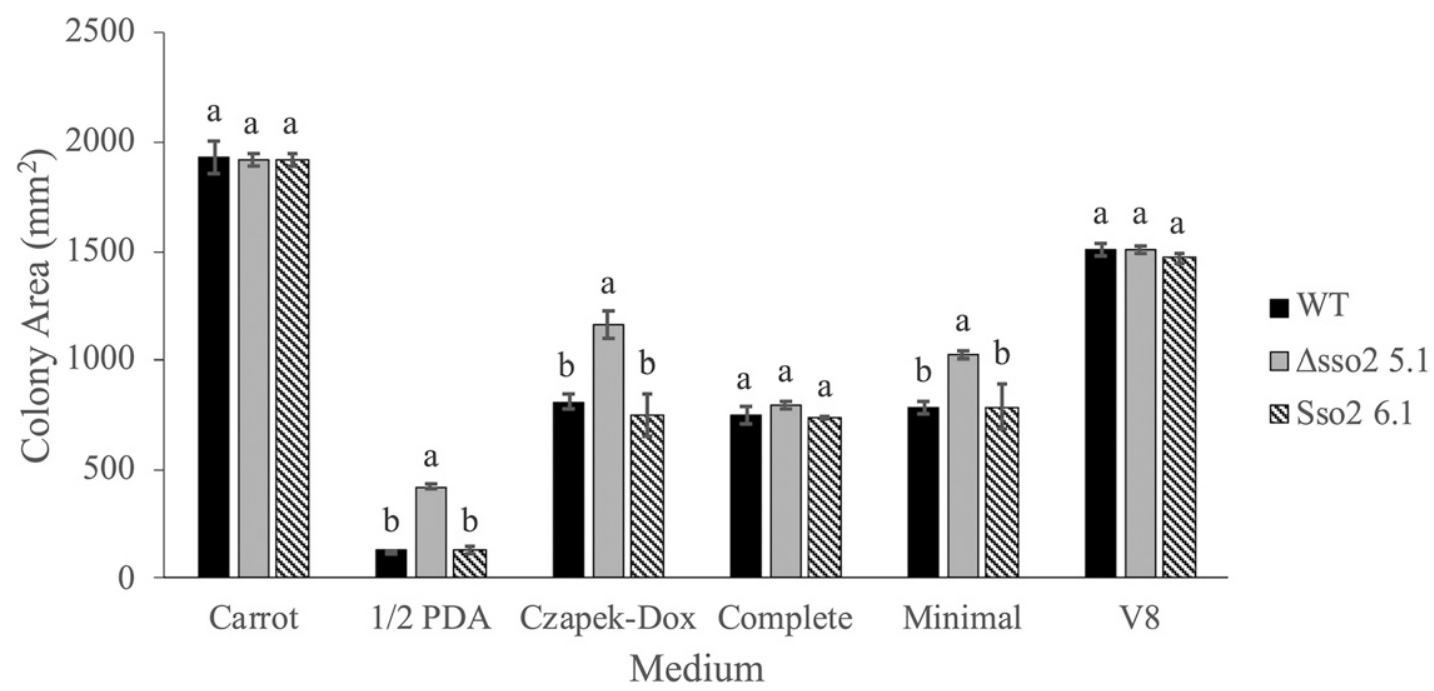

Fig. 2. Colony area of Fusarium graminearum $\Delta s s o 2$ mutant. Mean \pm standard deviation $(n=3)$ of the colony area of the $\Delta s s o 2$ mutant and ectopic transformant on six kinds of media. Significant differences between genotypes were determined by analysis of variance and letters indicate significance grouping. 
was found to localize to the subapical plasma membrane and septa, with a fainter RFP signal seen near the growing hyphal tips (Supplementary Fig. S5). This result would suggest that Sso2 is involved in the subapical endocytic membrane system.

The formation of subapical bulbous cells is a distinct morphological characteristic of DON-producing hyphae (Menke et al. 2012). For this reason, and to determine if Sso2-mediated export was involved in bulbous cell formation, a $\Delta s s o 2$ mutant was grown in toxin-inducing conditions and the number of bulbous cells formed were compared with wild type. To determine the potential effect of the mutation on Tri12-mediated export, the $\Delta s s o 2$ deletion mutant was generated in a Tri12GFP expressing background strain. The $\Delta$ sso 2 deletion mutant showed significantly fewer bulbous cells than either the Tri12GFP strain or wild type (Fig. 5). This suggests that $S s o 2$ may be involved in subapical membrane accumulation through vesicular exocytosis. However, no obvious difference was observed between wild type and the $\Delta s s o 2$ mutant for Tri12-GFP localization, vesicular content, or vacuolar size (data not shown).

\section{In-vitro DON accumulation}

\section{of $\boldsymbol{F}$. graminearum deletion mutants.}

To assess the role of Sso2 in the export of DON in vitro, the $\Delta s s o 2$ mutant was grown for 6 days in noninducing medium (NIM) or toxin induction medium (TBI) and was assessed for DON + ADON derivative accumulation. In NIM, there was little or no DON accumulation (typically $<1 \mathrm{ppm}$ ) and no significant differences compared with wild type $(P>0.05)$ (data not shown). In TBI, the $\Delta$ sso 2 mutant accumulated significantly less DON than the wild type, amassing roughly one fourth the amount of DON as the wild type (Fig. 6). Crossing the $\Delta s s o 2$ and $\Delta a b c 1$ deletion strains to generate a $\Delta a b c 1 / \Delta s s o 2$ double deletion mutant did not significantly alter the DON accumulation in vitro compared with $\Delta s s o 2$ alone.

\section{In-planta FHB symptoms and DON accumulation of $\boldsymbol{F}$. graminearum deletion mutants.}

To assess the role of Sso2 in the spread of FHB symptoms and DON accumulation in planta, the $\Delta s s o 2$ mutant was inoculated into wheat florets at anthesis and was allowed to grow for 14 days. Plants inoculated with the $\Delta s s o 2$ mutant accumulated significantly less DON after 14 days compared with wild type. DON accumulation was even further reduced in the $\Delta a b c 1 / \Delta s s o 2$ double deletion, compared with wild type and the individual single-deletion mutants (Fig. 7A), indicating an additive effect of $\Delta a b c 1$ and $\Delta s s o 2$ on reduction in DON accumulation during infection. Wheat heads were also analyzed for FHB symptoms. The $\Delta s s o 2$ mutant was significantly reduced in symptoms compared with wild type (Fig. 7B). The $\Delta a b c 1 / \Delta s s o 2$ mutant was significantly less virulent than wild type and the $\Delta$ sso2 single mutant but, although numerically reduced, was not significantly different (adjusted $P=0.55$ ) than that of the single $\triangle a b c l$ deletion mutant (Fig. 7A).

\section{Transcriptional impact \\ of $\boldsymbol{F}$. graminearum deletion mutants.}

To assess if the $\Delta s s o 2$ and $\Delta a b c l$ deletion mutations act directly to reduce DON accumulation by way of reducing export or perhaps indirectly by altering gene expression, total RNAs were extracted from wild type, $\Delta s s o 2$, and $\Delta a b c 1 F$. graminearum grown in TBI and analyzed via RNA-seq analysis. In total, there were 2,306 differentially expressed genes (DEGs) in the $\Delta$ sso 2 mutant and 224 DEGs in the $\Delta a b c 1$ mutant. Gene ontology (GO) enrichment analysis was conducted comparing the deletion mutants to wild type. The GO categories most significantly enriched among genes upregulated in the $\Delta$ sso 2 mutant included DNA recombination/repair, cellular response to DNA damage, and protein binding. The most significantly enriched GO categories for genes downregulated in the $\Delta$ sso2 mutant included oxidoreductase activity, redox processes, and catalytic/metabolic activity (Table 1). Nine trichothecene biosynthetic genes were downregulated in the $\triangle$ sso 2 mutant in addition to CLM1 and CLM2, the genes for the synthesis of the sesquiterpene mycotoxin culmorin. Also, in the biosynthetic gene cluster for the nonribosomal peptide synthase (NRPS) mycotoxin gramillin, 11 of 16 genes were significantly downregulated in the $\Delta$ sso 2 mutant. In contrast, many known NRPS and polyketide synthase (PKS) genes were up-regulated in the $\Delta s s o 2$ mutant including those for the estrogenic secondary metabolite zearalenone (Table 2). There were no GO categories significantly upregulated in the $\Delta a b c 1$ deletion mutant, and only three GO categories were downregulated, including redox processes and phospholipid binding. In addition, none of the trichothecene biosynthetic cluster genes or other sesquiterpene biosynthetic genes were significantly altered in expression compared with wild type (data not shown).

\section{DISCUSSION}

Two major plasma membrane-localized t-SNARE proteins have been described in filamentous fungi. One t-SNARE is enriched at the hyphal tip, in which it participates in normal hyphal-tip expansion, whereas the second t-SNARE is enriched at the subapical plasma membrane, in which it appears to direct lateral hyphal expansion, branching, and septum formation (Read 2011). The genes for these t-SNARES

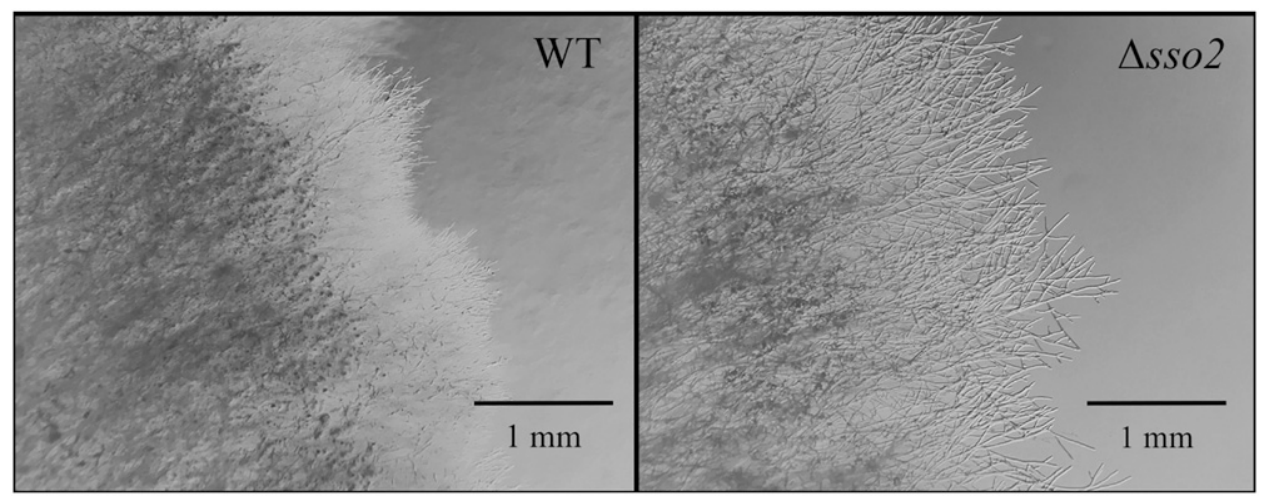

Fig. 3. Analysis of hyphal growth of the $\Delta s s o 2$ mutant. Representative images of wild type and $\Delta s s o 2$ mutant growth on half-strength potato dextrose agar. The $\Delta s s o 2$ mutant appears to branch less and to form a less-dense colony than wild-type Fusarium graminearum. 
are phylogenetically distinct paralogs (Kienle et al. 2009), but the naming conventions for the proteins in different fungi has led to confusion between the two. The t-SNARE generally found at the hyphal tip has been called Sso1 in Aspergillus spp. (Kuratsu et al. 2007), Magnaporthe spp. (Giraldo et al. 2013), and $F$. graminearum (Li et al. 2017), whereas the paralogous, subapically localized t-SNARE has been called Sso2. In
Neurospora spp. as well as in $F$. graminearum, these proteins have been called SYN1 and SYN2 (Gupta and Heath 2002; Hong et al. 2010). In Trichoderma spp., the naming convention has been reversed, with the hyphal-tip protein called Sso2 and the subapical form Sso1 (Valkonen et al. 2007). To be consistent with the naming convention in Aspergillus spp., Magnaporthe spp., and the designation of $\mathrm{Li}$ et al. (2017) for F. graminearum, we
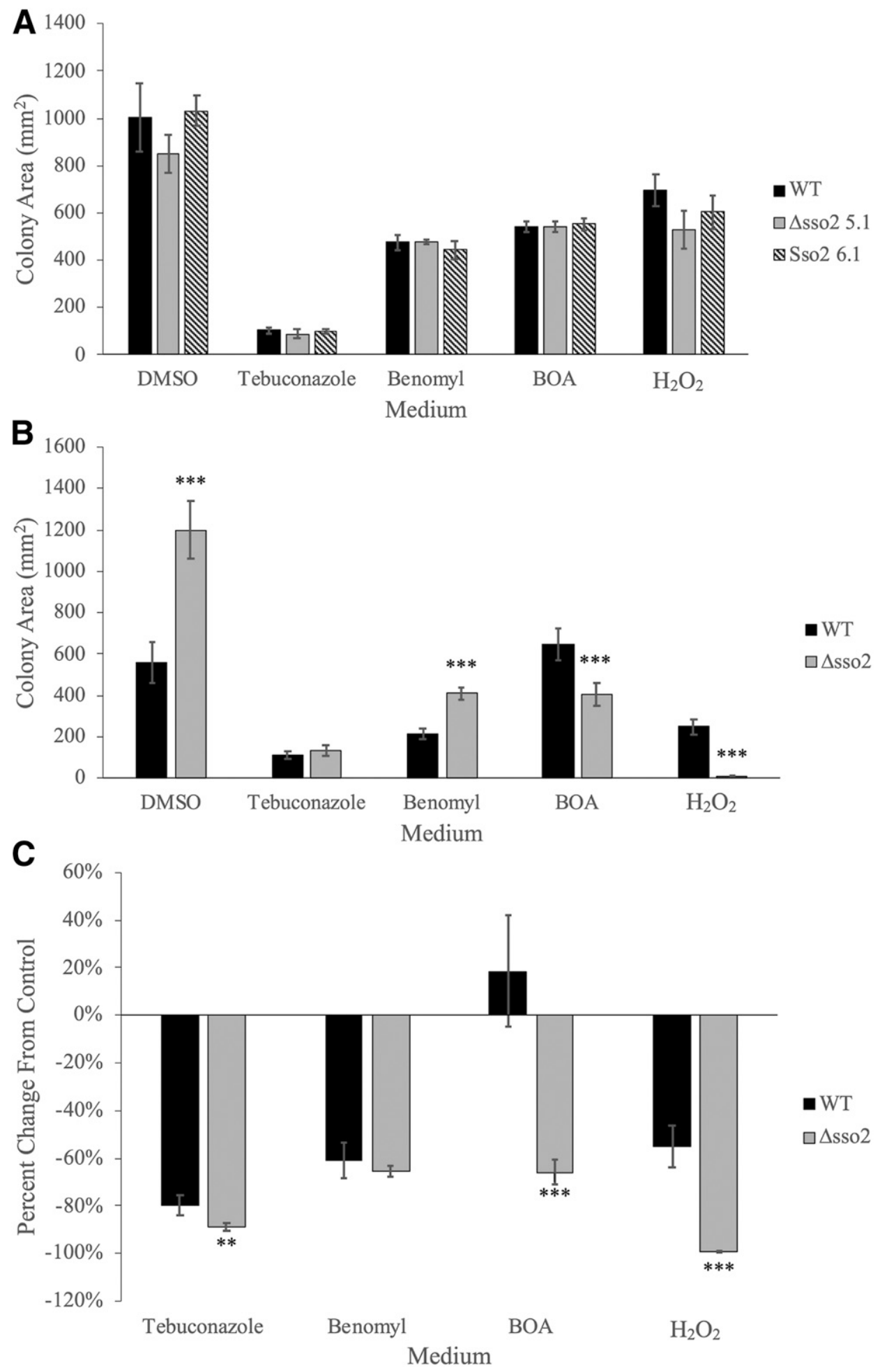

Fig. 4. Phenotypic response of Fusarium graminearum $\Delta s s o 2$ mutant. A, Response of $\Delta s s o 2$ mutant to exogenously applied xenobiotics on complete medium $(n=3)$ and $\mathbf{B}$ and $\mathbf{C}$, half-strength potato dextrose agar $(n=6)$. Values shown are means \pm standard deviation. Dimethyl sulfoxide acts as a carrier solvent for tested xenobiotics and is used as a control for those tests. BOA $=2$-benzoxazolinone. Significant differences were determined by Student's $t$ test and are indicated by asterisks; two asterisks $(* *)$ indicate $P<0.01$, three $(* * *)$ indicate $P<0.001$. 
have chosen to name the apical form Sso1 (FGSG_00950), while designating the t-SNARE studied here (FGSG_09928) as Sso2.

\section{Growth and phenotype of the $\Delta$ sso 2 mutant.}

The $\Delta s s o 2$ mutation influences the growth of $F$. graminearum on certain agar media but not others (Fig. 1). These findings expand upon the observations of Hong et al. (2010) that growth of a $\Delta$ sso 2 mutant (called $\Delta$ gzsyn 2 ) of $F$. graminearum GZ03643 was similar to that of wild type when grown on MM, carrot agar, and yeast malt agar medium. Like Hong et al. (2010), we found the $\Delta$ sso 2 mutant did not grow at a significantly different rate than wild type on carrot agar. However, we show here that $\Delta s s o 2$ does grow differently than wild type on $1 / 2$ PDA, CD, and MM, indicating that altered growth rate is dependent on the particular growth medium (Fig. 2). The mutant phenotype is unusual because the mutant has a greater radial growth rate than wild type on a variety of media. However, the $\Delta s s o 2$ mutant does not accumulate significantly greater biomass than wild-type F. graminearum when grown in liquid $1 / 2$ PDB for 3 days. Notably, the $\Delta$ sso 2 mutant appears to form fewer branches than wildtype $F$. graminearum when grown on PDA medium (Fig. 3). Taken together, this suggests that the increase in radial growth of the $\Delta$ sso2 mutant seen on certain agar media may be the result of the
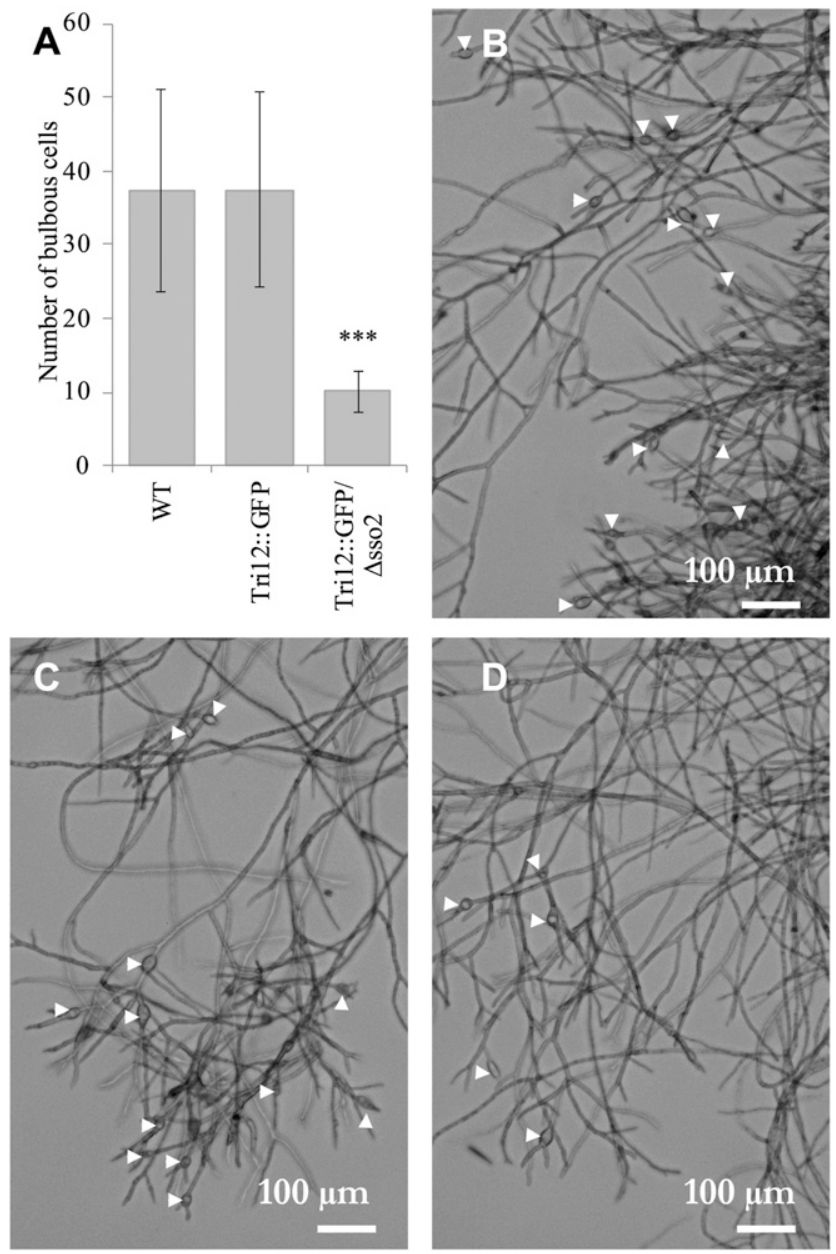

Fig. 5. Bulbous cell formation of a $\Delta s s o 2$ mutant. A, A $\Delta s s o 2$ deletion in a Tri12-GFP strain showed significantly fewer bulbous cells when grown in toxin-induction medium (mean \pm standard deviation of nine randomly selected fields of view). B, Representative micrographs of wild-type (WT) Fusarium graminearum, C, Tri12-GFP, and D, Tri12-GFP/Asso2. White arrows indicate the presence of a subapical bulbous cell. Significant differences were determined by Student's $t$ test. Three asterisks (***) indicate $P<0.001$. mutant forming fewer lateral branches, allowing for greater radial extension of advancing hyphae.

On $1 / 2$ PDA and CD, the $\Delta s s o 2$ genotype also did not produce the typical red pigmentation of the wild-type $F$. graminearum (Fig. 1). Interestingly, the $\Delta s s o 2$ mutant acidifies the medium to a significantly greater extent than did wild type. It has been shown that the same cultures of Fusarium species may appear orange-yellow in an acidic environment or red in a more alkaline environment (Ashley et al. 1937). Furthermore, mycelium of the wild-type $F$. graminearum appears red in NIM but is yellow in TBI, where it is known to acidify its environment (Gardiner et al. 2009b). Low pH $(\leq 4)$ has been shown to inhibit the production of the red pigment aurofusarin in F. graminearum (Medentsev et al. 2005). However, alkalization of the $\Delta$ sso 2 containing $1 / 2$ PDB medium to the range seen in the wild type did not restore red pigmentation, perhaps suggesting an altered pigment production rather than a $\mathrm{pH}-$ induced color change (unpublished data).

The $\Delta$ sso 2 mutant may be altered in the synthesis or export of its major red pigment, aurofusarin. Previous research has indicated that the metabolism of the yellow precursor hydroxyrubrofusarin to aurofusarin is catalyzed extracellularly by the oxidoreductase AurO (Frandsen et al. 2011) and deletion of the predicted aurofusarin transporter AurT did not result in any detectable differences in the aurofusarin pathway or pigment production (Frandsen et al. 2011; Kim et al. 2008). While most of the biosynthetic genes associated with aurofusarin production were unchanged in the $\Delta s s o 2$ mutant, the oxidoreductase AurO was significantly upregulated (Table 2). It may be possible

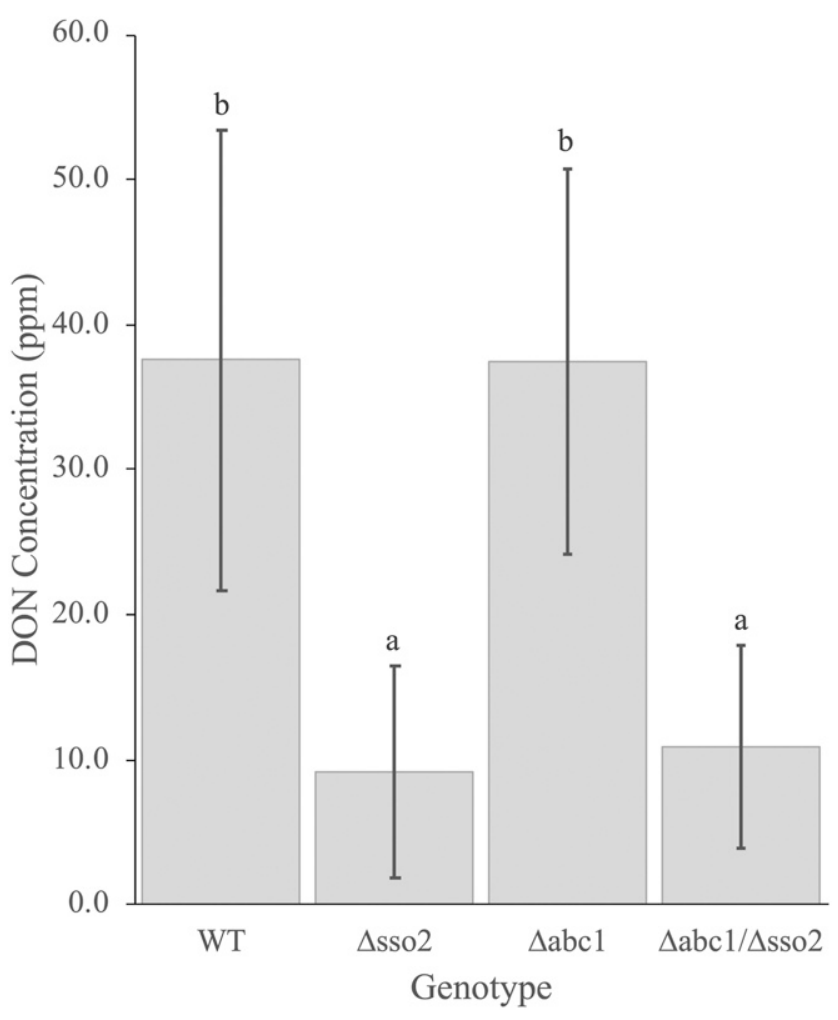

Fig. 6. In vitro deoxynivalenol (DON) accumulation of $\Delta s s o 2$ and $\Delta a b c 1$ mutants. Accumulation of DON + ADON derivatives in vitro after 6 days of growth in toxin-induction medium $(n=15)$, shown as mean \pm standard deviation. The $\Delta a b c 1$ mutant was not significantly different from wild type, while the $\Delta s s o 2$ mutant was significantly reduced in DON accumulation. The double mutant $\Delta a b c 1 / \Delta s s o 2$ is not significantly reduced in DON accumulation in vitro compared with $\Delta s s o 2$ alone, suggesting no additive effect. Significant differences between genotypes were determined by analysis of variance and letters indicate significance groupings. 
that either the precursor hydroxyrubrofusarin, the enzyme AurO used to synthesize aurofusarin, or both, are packaged in vesicles destined for docking to the t-SNARE Sso2. In the absence of the proper docking site, the precursor and AurO may not be exported and so the extracellular red pigment may not accumulate. However, initial sodium dodecyl sulfate-polyacrylamide gel electrophoresis gel comparison of total extracellular proteins from the $\Delta s s o 2$ mutant grown in TBI did not show discernable differences in banding patterns compared with wild type (Supplementary Fig. S6). A more detailed examination of the extracellular proteome will be required to address this hypothesis.

When grown on CM supplemented with xenobiotics, there were no significant differences in the growth of the $\Delta s s o 2 \mathrm{mu}-$ tant or an ectopic transformant, as compared with wild type, indicating no increased sensitivity to tested compounds (Fig. 4A). However, the $\Delta$ sso 2 mutant was shown to be sensitive to the xenobiotics tebuconazole, BOA, and hydrogen peroxide when grown on 1/2 PDA (Fig. 4B). This suggests that Sso2mediated membrane fusion plays an important, yet culturedependent, role in the resistance of $F$. graminearum to fungicidal or inhibitory compounds. Interestingly, BOA and oxidative stress caused by $\mathrm{H}_{2} \mathrm{O}_{2}$ are likely to occur naturally in planta when F. graminearum infects wheat (Desmond et al. 2008; Villagrasa et al. 2006). The sensitivity of the $\Delta$ sso 2 mutant to these compounds indicates that this mutant may be less aggressive during infection due to increased sensitivity to plant defenses.

When screened against a wider array of xenobiotics, using the Biolog microarray system, the $\Delta$ sso 2 mutant showed increased sensitivity to many tested compounds (Supplementary Fig. S3). Some compounds showed little to no inhibitory effect against wild-type $F$. graminearum but showed inhibitory action against the $\Delta s s o 2$ mutant. This finding indicates that Sso2mediated membrane fusion plays an important role in the defense against a wide array of xenobiotics and its absence causes sensitivity to many classes of compounds. While much less frequent, there were a few compounds on which the $\Delta$ sso 2 mutant grew better. Increased resistance to neomycin and paromomycin likely resulted from the neomycin-resistance construct used to generate the $\Delta$ sso 2 mutant.

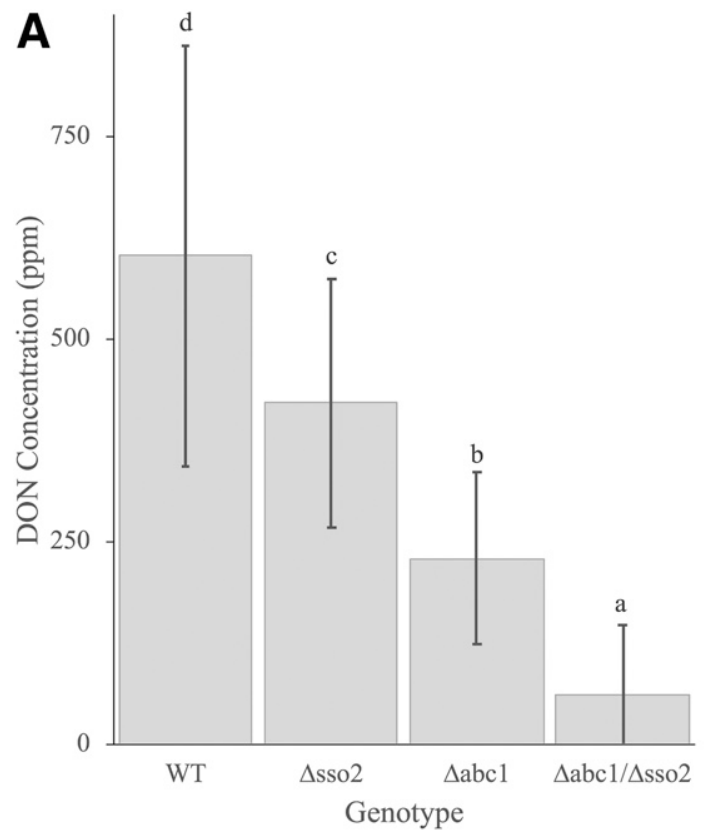

In a separate carbon-utilization experiment using the Biolog SPF2 plate, the $\Delta s s o 2$ mutant showed decreased growth on many mono- and disaccharide compounds (Supplementary Fig. S4). This suggests that Sso2 may play an important role in either endocytic transport of sugar compounds, transport the enzymes that cleave the glycosidic linkages within disaccharides (e.g., glycoside hydrolases), or both. This may also help explain the differences in growth and the increased xenobiotic sensitivity of the $\Delta s s o 2$ mutant on $1 / 2$ PDA, since Sso 2 may be involved in nutrient uptake, utilization, or other endocytic processes, such as xenobiotic processing or sequestration, on this complex medium. For comparison, CM is a defined medium containing glucose as sole saccharide, whereas 1/2 PDA is a rich medium containing potato extract likely containing many complex oligo- and polysaccharides. If Sso2 facilitates one or both the nutrient uptake of sugars and export of enzymes involved in cleaving glycosidic linkages, growth defects might be more severe on a rich medium containing these compounds than on a defined medium that does not.

\section{Localization of an RFP-tagged Sso2.}

When grown in PDB for $30 \mathrm{~h}$, a Sso2-RFP-tagged strain showed that the Sso 2 protein localized to the subapical plasma membrane and septa (Supplementary Fig. S5). This finding confirms results of previous localization experiments that identified Sso2 at the same location of mature hyphae (Hong et al. 2010). In addition, the $\Delta s s o 2$ mutant showed reduced subapical bulbous cell formation (Fig. 5), a distinct characteristic of DON-producing cells (Menke et al. 2012). Taken together, these results suggest that Sso2 may either play a role in subcellular reorganization of toxigenic cells, may be involved directly in DON export, or both.

\section{In-vitro DON accumulation \\ of $\boldsymbol{F}$. graminearum deletion mutants.}

When the $\Delta$ sso 2 mutant was grown under DON noninducing conditions (NIM), there was very little trichothecene accumulation (typically less than $1 \mathrm{ppm}$ ). This is expected, as NIM contains sodium nitrate as the sole nitrogen source and does not

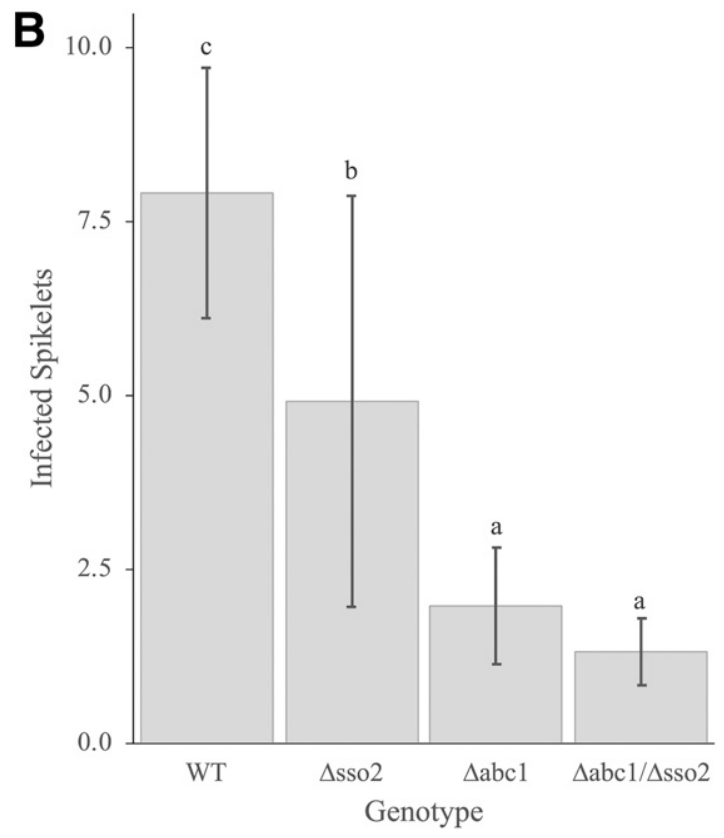

Fig. 7. In planta virulence and deoxynivalenol (DON) accumulation of $\Delta s s o 2$ and $\Delta a b c 1$ mutants. A, DON+ADON accumulation and B, virulence 14 days postinoculation $(n=25)$, shown as mean \pm standard deviation. Virulence is a measure of infected florets $(\leq 10)$ after 14 days. All mutant genotypes are reduced in DON accumulation and virulence compared with wild type. The $\Delta a b c 1 / \Delta s s o 2$ mutant shows an additive reduction in DON accumulation but not virulence compared with the single deletion genotypes $\Delta a b c 1$ and $\Delta s s o 2$. Different letters indicate significant differences groupings. 
contain any other nitrogen sources known to induce DON production (Gardiner et al. 2009a). When grown in DON inducing conditions (TBI), DON accumulation in the $\Delta$ sso2 mutant was significantly reduced compared with wild type (Fig. 6). Combining the $\Delta s s o 2$ mutation with the $\Delta a b c 1 \mathrm{mu}-$ tation did not significantly reduce the accumulation of DON in TBI, as compared with $\Delta s s o 2$ alone. The $\Delta a b c l$ mutant alone is not significantly reduced in DON accumulation compared with wild type under these conditions and, in fact, our RNA-seq data indicate the gene is expressed only at very low levels in vitro. Thus, stacking this deletion with the already significantly reduced $\Delta s s o 2$ mutant may not be expected to have a large effect in culture.

\section{In-planta DON accumulation and virulence of $F$. graminearum mutants.}

When assessed in planta for FHB symptoms, deletion of Sso2 reduced both DON accumulation and virulence by nearly $50 \%$. Additionally, the $\Delta s s o 2$ mutant was significantly reduced in bulbous cell formation. Bulbous cells form during toxininducing conditions (Menke et al. 2012), even for mutants deficient in DON accumulation (Jonkers et al. 2012), suggesting that their formation, while being a hallmark of toxigenic cells, is not a direct consequence of the synthesis or accumulation of DON. Nevertheless, these findings indicate that Sso2mediated subapical membrane fusion may be an important mechanism contributing to $F$. graminearum virulence, DON export, and subapical bulbous cell formation. Previous research has indicated the $F$. graminearum SNARE protein FgVam7 is involved in virulence and DON accumulation; FgVam7 appears to form a complex with the apical SNARE FgSsol (GzSYN1; FGSG_00950; FGRAMPH1_01G02393), and deletion of FgVam7 significantly reduces polar growth (Li et al. 2017; Zhang et al. 2016), suggesting its role in apical membrane fusion. However, our work is the first report of the importance of the Sso2mediated membrane fusion as a major route of DON export both in vitro and in planta.

Combining the $\Delta s s o 2$ and $\Delta a b c 1$ mutations in the same strain reduced DON accumulation in an additive manner in planta compared with the single mutation strains. DON accumulation was significantly reduced in the $\Delta a b c 1 / \Delta$ sso 2 mutant compared with wild type and both of its parent genotypes (Fig. 7A). However, the combined $\Delta a b c 1 / \Delta s s o 2$ strain did not significantly reduce the virulence of $F$. graminearum compared with the $\Delta a b c 1$ deletion mutant alone (Fig. 7B). Indeed, the $\Delta a b c 1$ mutant was already near the minimum virulence measurable by this assay, and, as a result, adding the $\Delta s s o 2$ mutation to this background could not further reduce virulence much more than the $\Delta a b c l$ mutant alone. Taken together, this suggests that deleting multiple export pathways in $F$. graminearum can significantly reduce DON accumulation in planta, potentially further reducing contamination of grains with potent toxins.

An important difference to note between the in vitro and in planta results is the magnitude of DON accumulation between the two tests. In-vitro tests typically result in a maximum DON concentration of approximately $50 \mathrm{ppm}$ (Fig. 6), while inplanta DON accumulation of wild type can surpass $500 \mathrm{ppm}$ (Fig. 7A). The fact that the $\Delta a b c 1$ mutant failed to show reduced DON accumulation in vitro but did show reduced DON accumulation in planta could result from differences in expression levels under these two conditions. While $A b c l$ transcripts are barely detectable in culture, the gene is greatly upregulated during wheat infection (Lysøe et al. 2011; Wang et al. 2017). Additionally, other membrane-bound transporters,

Table 1. Most significantly enriched differentially expressed gene (DEG) ontology (GO) categories in the $\Delta s s o 2$ mutant $^{\mathrm{a}}$

\begin{tabular}{|c|c|c|c|}
\hline GO term & Category $^{\mathbf{b}}$ & Bonferroni $P$ value & Enrichment ratio \\
\hline \multicolumn{4}{|c|}{ The top 15 enriched GO terms of upregulated DEGs in $\Delta s s o 2$ versus PH1 } \\
\hline DNA recombination & BP & $1.03 \mathrm{E}-06$ & 0.53 \\
\hline DNA repair & $\mathrm{BP}$ & $1.48 \mathrm{E}-06$ & 0.3 \\
\hline Cellular response to DNA damage stimulus & $\mathrm{BP}$ & 2.24E-06 & 0.28 \\
\hline Binding & MF & $2.27 \mathrm{E}-05$ & 0.11 \\
\hline Phosphopantetheine binding & MF & $5.07 \mathrm{E}-05$ & 0.5 \\
\hline Small molecule binding & MF & 7.24E-05 & 0.14 \\
\hline DNA metabolic process & $\mathrm{BP}$ & $9.31 \mathrm{E}-05$ & 0.23 \\
\hline Anion binding & MF & 0.000123219 & 0.14 \\
\hline Modified amino acid binding & MF & 0.000153111 & 0.46 \\
\hline Structural constituent of cytoskeleton & MF & 0.00021363 & 0.59 \\
\hline Neuron projection guidance & $\mathrm{BP}$ & 0.000411833 & 0.88 \\
\hline Adenyl nucleotide binding & MF & 0.000984349 & 0.15 \\
\hline Nucleoside phosphate binding & MF & 0.001081363 & 0.13 \\
\hline Nucleotide binding & MF & 0.001081363 & 0.13 \\
\hline Cellular response to stress & $\mathrm{BP}$ & 0.001130939 & 0.21 \\
\hline \multicolumn{4}{|c|}{ The top 15 enriched GO terms of downregulated DEGs in $\Delta s s o 2$ versus PH1 } \\
\hline Oxidoreductase activity & MF & $9.54 \mathrm{E}-24$ & 0.14 \\
\hline Oxidation-reduction process & $\mathrm{BP}$ & $2.56 \mathrm{E}-21$ & 0.14 \\
\hline Catalytic activity & MF & $9.50 \mathrm{E}-19$ & 0.09 \\
\hline Metabolic process & $\mathrm{BP}$ & $1.23 \mathrm{E}-08$ & 0.08 \\
\hline Cofactor binding & MF & 4.19E-06 & 0.12 \\
\hline Biological process & $\mathrm{BP}$ & $5.78 \mathrm{E}-06$ & 0.07 \\
\hline Transmembrane transport & $\mathrm{BP}$ & 0.000186393 & 0.11 \\
\hline Coenzyme binding & MF & 0.000931845 & 0.12 \\
\hline Organic acid metabolic process & $\mathrm{BP}$ & 0.001567231 & 0.12 \\
\hline Oxoacid metabolic process & $\mathrm{BP}$ & 0.00244007 & 0.12 \\
\hline Sulfur compound metabolic process & $\mathrm{BP}$ & 0.005747767 & 0.21 \\
\hline Small molecule metabolic process & $\mathrm{BP}$ & 0.013655861 & 0.1 \\
\hline Fatty acid synthase activity & MF & 0.025446468 & 0.8 \\
\hline Nitrogen cycle metabolic process & $\mathrm{BP}$ & 0.025790733 & 0.63 \\
\hline Iron ion binding & MF & 0.026183384 & 0.13 \\
\hline
\end{tabular}

${ }^{a}$ Enrichment of gene ontology categories as performed with the Gene Ontology Enrichment tool in FungiDB (Basenko et al. 2018).

${ }^{\mathrm{b}} \mathrm{BP}=$ biological process, $\mathrm{MF}=$ molecular function, and $\mathrm{CC}=$ cellular component . 
such as the trichothecene efflux transporter Tril2 (Menke et al. 2012) or other transporters, could be compensating for $\mathrm{Abcl}$ when it is deleted. A previous analysis indicated that $F$. graminearum has 62 potential $\mathrm{ABC}$ transporters in its genome (Kovalchuk and Driessen 2010), some of which may have functional redundancy. Nevertheless, since DON concentrations are much higher in planta, when Abc1 is not available for DON export in the $\Delta a b c l$ mutant, other

Table 2. Differential gene expression of important secondary metabolite gene clusters ${ }^{\mathrm{a}}$

\begin{tabular}{|c|c|c|c|}
\hline Gene ID & Comments & Description & Fold change $^{b}$ \\
\hline \multicolumn{4}{|c|}{ Trichothecene (TRI) biosynthetic genes } \\
\hline FGRAMPH1_01G00223 & TRI1 & Trichothecene C-8 oxygenase & -2.367 \\
\hline FGRAMPH1_01G13105 & TRI3 & Trichothecene C- 15 acetyltransferase & -2.6 \\
\hline FGRAMPH1_01G13107 & TRI4 & Trichodiene oxygenase & -2.616 \\
\hline FGRAMPH1_01G13111 & TRI5 & Trichodiene synthase & -3.241 \\
\hline FGRAMPH1_01G13109 & TRI6 & Transcription factor & -1.588 \\
\hline FGRAMPH1_01G13103 & TRI7 & Trichothecene C-3 acetyltransferase & -1.656 \\
\hline FGRAMPH1_01G13101 & TRI8 & Trichothecene C-3 esterase & -2.044 \\
\hline FGRAMPH1_01G13115 & TRI9 & Unknown function & -1.872 \\
\hline FGRAMPH1_01G13113 & TRI10 & Transcription factor & -1.729 \\
\hline FGRAMPH1_01G13117 & TRI11 & Trichothene C-15 hydroxylase & -2.228 \\
\hline FGRAMPH1_01G13119 & TRI12 & Trichothecene efflux pump & -2.308 \\
\hline FGRAMPH1_01G13121 & TRI13 & Trichothecene C-3 oxygenase & -2.685 \\
\hline FGRAMPH1_01G13123 & TRI14 & Unknown function & -2.797 \\
\hline FGRAMPH1_01G21051 & TRI15 & Putative zinc finger & -1.305 \\
\hline FGRAMPH1_01G25923 & TRI101 & Trichothecene 3-O-acetyltransferase & -1.963 \\
\hline \multicolumn{4}{|l|}{ Sesquiterpene synthesis genes } \\
\hline FGRAMPH1_01G13111 & TRI5 & Trichodiene synthase & -3.241 \\
\hline FGRAMPH1_01G07999 & CLM1 & Longiborneol synthase & -2.804 \\
\hline FGRAMPH1_01G00053 & CLM2 & Longiborneol oxygenase & -2.472 \\
\hline FGRAMPH1_01G22293 & & Hypothetical protein & -1.161 \\
\hline FGRAMPH1_01G13013 & & Aristolochene synthase & -1.179 \\
\hline FGRAMPH1_01G09299 & & Presilphiperfolan-8-beta-ol synthase & $2,069.854$ \\
\hline FGRAMPH1_01G21787 & & Hypothetical protein & 1.124 \\
\hline FGRAMPH1_01G01167 & & Hypothetical protein & 1.083 \\
\hline FGRAMPH1_01G25349 & & Terpene synthase metal-containing & 1.398 \\
\hline \multicolumn{4}{|l|}{ Gramillins (NRPS8) cluster } \\
\hline FGRAMPH1_01G00127 & & Fatty acid synthase alpha partial & -2.702 \\
\hline FGRAMPH1_01G00129 & & Cytochrome E- group I & -2.683 \\
\hline FGRAMPH1_01G00131 & & Fatty acid synthase beta subunit & -2.764 \\
\hline FGRAMPH1_01G00133 & & Unnamed protein product & -1.768 \\
\hline FGRAMPH1_01G00135 & & Hypothetical protein & -1.198 \\
\hline FGRAMPH1_01G00137 & & Unc-45 like B & -1.228 \\
\hline FGRAMPH1_01G00139 & & Nitrogen catabolic enzyme regulatory & -1.504 \\
\hline FGRAMPH1_01G00141 & GRA2 & Myc-type basic helix-loop-helix domain & -1.867 \\
\hline FGRAMPH1_01G00143 & GRA1/NRPS8 & Nonribosomal peptide synthetase & $-\mathbf{4 . 0 7}$ \\
\hline FGRAMPH1_01G00145 & & Thioredoxin reductase & $-\mathbf{3 . 0 5 3}$ \\
\hline FGRAMPH1_01G00147 & & Hydrolase or acyltransferase of $\alpha / \beta$ superfamily & -2.725 \\
\hline FGRAMPH1_01G00149 & & $\alpha / \beta$ Hydrolase fold -1 & -2.271 \\
\hline FGRAMPH1_01G00151 & & Hypothetical protein & -2.264 \\
\hline FGRAMPH1_01G00153 & & Hypothetical protein & -2.727 \\
\hline FGRAMPH1_01G00155 & & Oxoglutarate iron-dependent oxygenase & -2.311 \\
\hline FGRAMPH1_01G00157 & & Branched-chain-amino-acid aminotransferase & -2.833 \\
\hline \multicolumn{4}{|c|}{ Zearalenone (PKS4/PKS13) cluster } \\
\hline FGRAMPH1_01G05751 & PKS4 & Polyketide synthase (PKS) & 2.158 \\
\hline FGRAMPH1_01G05749 & PKS13 & PKS & 27.358 \\
\hline FGRAMPH1_01G05753 & ZEB1 & FAD binding domain-containing & 3.08 \\
\hline FGRAMPH1_01G05755 & ZEB2 & Hypothetical protein & -1 \\
\hline \multicolumn{4}{|l|}{ Aurofusarin (Aur) cluster } \\
\hline FGRAMPH1_01G05585 & AurR1 & Positive acting transcription factor & 1.474 \\
\hline FGRAMPH1_01G05587 & AurO & Oxidoreductase & 2.611 \\
\hline FGRAMPH1_01G05589 & AurT & Major facilitator transporter & 1.406 \\
\hline FGRAMPH1_01G05591 & AurR2 & Transcription factor & 1.548 \\
\hline FGRAMPH1_01G05593 & PKS12 & Aurofusarin PKS & 1.995 \\
\hline FGRAMPH1_01G05595 & AurZ & Conidial pigment PKS partial & 2.304 \\
\hline FGRAMPH1_01G05597 & AurJ & Sterigmatocystin 8-o-methyltransferase & 2.156 \\
\hline FGRAMPH1_01G05599 & AurF & Flavin monooxygenase & 2.251 \\
\hline FGRAMPH1_01G05601 & Gip1 & Laccase & 2.171 \\
\hline FGRAMPH1_01G05603 & AurS & Fasciclin domain family & 1.765 \\
\hline
\end{tabular}

\footnotetext{
a Many secondary metabolite biosynthetic genes, particularly those involved in deoxynivalenol synthesis are downregulated compared with wild type. Gene expression of presilphiperfolan-8 $\beta$-ol (predicted), zearalenone, and aurofusarin synthesis is unchanged or upregulated.

b The $\Delta s s o 2$ strain versus wild type (WT) fold change. Values in bold are downregulated with fold change $<-2$ and false discovery rate (FDR) $<0.05$; values in bold and italics are upregulated with fold change $>2$ and FDR $<0.05$.
} 
transporters may not be able to fully compensate. The same may not be true for Sso2, which showed similar reductions in DON accumulation both in vitro and in planta, suggesting a more integral, irreplaceable role in DON accumulation.

Our results on the importance of Abc1 in virulence to wheat and DON accumulation by $F$. graminearum fit into a larger picture forming of the role of this transporter. Deletion mutants of $A b c l$ show reduced virulence during wheat crown and rootrot infections (Gardiner et al. 2013). Similarly, strains of $F$. graminearum that produce DON or the closely related trichothecene nivalenol show reduced virulence on wheat, barley, and maize and altered mycotoxin production when $\mathrm{Abcl}$ (sometimes referred to as $A b c 3$ ) is deleted (Abou Ammar et al. 2013). Abc1 is also involved in transport of zearalenone, an estrogenic metabolite of $F$. graminearum, and tolerance to antifungal compounds (Abou Ammar et al. 2013; Gardiner et al. 2013; Lee et al. 2011). The exact function of Abc1 and other potential DON exporting transporters is currently being investigated. These transporters have been cloned into a DONsensitive yeast strain to assess their protective capabilities and confirm their activity toward trichothecenes (S. P. O'Mara and H. C. Kistler unpublished).

\section{Transcriptional impact}

of $\boldsymbol{F}$. graminearum deletion mutants.

In vitro, the $\Delta a b c l$ deletion mutant had a very small impact on the expression of other $F$. graminearum genes, particularly those of the trichothecene biosynthetic cluster. This may help explain the lack of an effect in DON accumulation of this mutant when grown in TBI. While Sso2 is a component of a vesicular transport system, deletion of the gene for this protein has a profound effect on the Fusarium transcriptome. The most significantly enriched GO categories of upregulated genes in the $\Delta$ sso 2 mutant included DNA recombination/repair, cellular response to DNA damage, and protein binding (Table 1). This suggests that the deletion of Sso 2 may be causing intracellular damage, potentially due to a buildup of the toxin DON. DON is a eukaryotic ribosome inhibitor (Garreau de Loubresse et al. 2014) and is also known to cause DNA/RNA synthesis disruption (Rocha et al. 2005). Deletion of Sso2 may inhibit F. graminearum from sequestering and exporting DON, causing intracellular damage that induces the expression of numerous repair mechanisms. The most significantly enriched GO categories of downregulated genes in the $\Delta s s o 2$ mutant included oxidoreductase activity, redox processes, and catalytic/metabolic activity. This suggests that many primary metabolic processes are being downregulated, potentially in response to the intracellular damage caused by a lack of DON sequestration and export.

Importantly, most of the trichothecene biosynthetic genes involved in DON synthesis were downregulated in the $\Delta s s o 2$ mutant (Table 2), including the sesquiterpene synthase gene that catalyzes the first step in trichothecene synthesis, Tri5, and the cluster-localized efflux pump, Tri12. The accumulation of mycotoxins culmorin and gramillin also are known to be coregulated with DON (Bahadoor et al. 2018; Flynn et al. 2019; Jonkers et al. 2012) in F. graminearum, and so it is noteworthy that the culmorin biosynthetic genes CLM1 and CLM2 and 11 of the 16 biosynthetic genes within the gramillin biosynthetic gene cluster were also significantly down-regulated in the $\Delta$ sso2 mutant. This suggests a feedback mechanism, slowing production of these known secondary metabolite virulence factors (Bahadoor et al. 2018; Maier et al. 2006; McCormick et al. 2010) when Sso2-mediated processes are eliminated.

In summary, the t-SNARE Sso2 appears to be important for normal growth of $F$. graminearum and defense against xenobiotics in a medium-dependent manner. Additionally, in the absence of Sso2, expression of genes for DON and numerous other secondary metabolite gene clusters are down-regulated and intracellular repair mechanisms are the most up-regulated gene category. Sso2 is also important for $\mathrm{pH}$ homeostasis and for the formation of bulbous cells, a morphological shift correlated with DON production, and plays a role in accumulation of DON both in vitro and in planta as well as for full FHB disease progression. Combining deletions of the $\mathrm{Sso} 2$ and $\mathrm{Abcl}$ genes caused significant reduction of DON accumulation in planta in an additive manner. These findings indicate that $\mathrm{Sso} 2$ and $A b c l$ may be potential targets for future genetic FHB management techniques, such as host-induced gene silencing (HIGS), especially when targeted together. Recent research has shown that HIGS can be a powerful tool in wheat resistance to pathogens and in FHB management (Cheng et al. 2015; Koch et al. 2016; Qi et al. 2019). Using genetic techniques to affect the expression of $S s o 2$ and $A b c 1$ can be a viable new avenue for FHB management, especially when combined with other management techniques such as fungicide application, crop rotation, and biocontrol.

\section{MATERIALS AND METHODS}

Generation of $\boldsymbol{F}$. graminearum single-deletion mutants.

Gene-deletion mutants of $F$. graminearum PH1 (NRRL 31084) were generated using the split marker homologous recombination technique (Goswami 2012). The 5' and 3' flanking regions of genes to be deleted were amplified, using the oligonucleotides listed in Supplementary Table S1. Flanking regions were approximately 800 to $1,200 \mathrm{bp}$ in length. The inward-facing primer of each primer pair (i.e., reverse primer for $5^{\prime}$ regions and forward primer for $3^{\prime}$ regions) contained an approximately 20-bp $5^{\prime}$ tail complementary to each end of the antibiotic resistance gene used to select for knockout mutants. The target gene was replaced with the neomycin phosphotransferase resistance gene from pSM334, as a selectable marker (Fuchs et al. 2004). A primary round of PCR amplification resulted in approximately 800- to 1,200-bp amplicons of the $5^{\prime}$ and $3^{\prime}$ flanks of the gene to be deleted. A secondary round of PCR amplification resulted in fusing the $5^{\prime}$ amplicon to the upstream two-thirds of the neomycin phosphotransferase resistance gene and fusing the $3^{\prime}$ amplicon to the downstream two-thirds of the same antibiotic resistance gene.

Transformations were performed as previously described (Menke et al. 2013). Three micrograms of each fusion PCR product and $1 \times 10^{8} \mathrm{~F}$. graminearum protoplasts were combined in $4 \mathrm{ml}$ of liquid TB3 $(0.3 \%$ yeast extract, $0.3 \%$ casamino acids, $0.6 \mathrm{M}$ sucrose) medium and were incubated on an orbital shaker at $150 \mathrm{rpm}$ and $25^{\circ} \mathrm{C}$. After overnight incubation, liquid TB3 containing $F$. graminearum protoplasts was combined with melted TB3 containing $0.7 \%$ low-melting point agarose and supplemented with $150 \mu \mathrm{g}$ of neomycin per milliliter and was aliquoted into six $10-\mathrm{cm}$ petri plates $(12.5 \mathrm{ml}$ each). Transformation plates were incubated overnight at $25^{\circ} \mathrm{C}$ in the dark. On the next day, $7.5 \mathrm{ml}$ of melted TB3 containing $250 \mu \mathrm{g}$ of neomycin per milliliter were overlaid on each of six plates, while one control plate without neomycin was used to confirm successful germination of protoplasts. Plates were incubated in the dark at $25^{\circ} \mathrm{C}$ for 7 to 10 days before picking antibioticresistant transformant colonies.

Up to 10 transformant colonies were picked and transferred onto a 6-cm petri plate with $10 \mathrm{ml}$ of V8 juice medium $(200 \mathrm{ml}$ of V8 juice [Campbell Soup Company, Camden, NJ, U.S.A.], $2 \mathrm{~g}$ of $\mathrm{CaCO}_{3}, 15 \mathrm{~g}$ of agar, and water to 1 liter) containing $150 \mu \mathrm{g}$ of neomycin per milliliter. V8 plates were incubated at $25^{\circ} \mathrm{C}$ for approximately 7 days. To isolate a single genotype of antibiotic-resistant transformant $F$. graminearum, a small amount of the aerial hyphae bearing conidia was transferred 
and dilution-plated on water agar containing $150 \mu \mathrm{g}$ of neomycin per milliliter. Dilution plates were incubated overnight at $25^{\circ} \mathrm{C}$, to allow time for germination. The following day, two germinated conidia were identified using a binocular dissecting microscope and were individually transferred onto a new 6-cm petri plate with $10 \mathrm{ml}$ of V8 juice medium containing $150 \mu \mathrm{g}$ of neomycin per milliliter. Single-spored plates were incubated at $25^{\circ} \mathrm{C}$ for approximately 7 days.

\section{Generation of $\boldsymbol{F}$. graminearum double-deletion mutants.}

Single-deletion mutants of $F$. graminearum were crossed to generate a double-deletion mutant. To select for the double knockout mutant, single knockout mutant parents contained different antibiotic-resistance selectable markers. A $\Delta a b c l$ (FGSG_04580; FGRAMPH1_01G15627) deletion mutant was generated using the same technique described earlier, except a hygromycin-resistance cassette rather than a neomycinresistance cassette was used. The $\Delta s s o 2$ (neoR) and a $\Delta a b c 1$ (hygR) (Lupton et al. 1991) single-deletion mutant were grown on the same carrot agar plate. Mutants were grown for 7 to 10 days, allowing for contact between the two genotypes. Afterward, total mycelium was knocked down using a $2.5 \%$ Tween-60 aqueous solution and mutants were incubated for another 7 to 10 days or until perithecia with ascospores had formed. Discharged ascospores adhere to the petri plate lids (Pasquali and Kistler 2006). To collect discharged ascospores, the lid on each petri plate was removed and washed with $1 \mathrm{ml}$ of sterile water to dislodge ascospores and the spore suspension was transferred to a sterile $1.5-\mathrm{ml}$ centrifuge tube. This process was repeated every few days to continually collect discharged ascospores.

Once collected, ascospores were pooled and were enumerated using a hemocytometer. Ascospore suspensions were diluted to approximately $2 \times 10^{4}$ spores per milliliter and were aliquoted onto 1/2 PDA plates with antibiotic selection, such that only recombinant ascospores containing both parental drug resistance markers would survive. Concentration of antibiotics was identical as for single resistance selection. Final concentration of ascospores on each plate ranged from 50 to 10,000 spores per plate to account for potentially differing frequencies of recombination events. After roughly one week, $<10$ separate colonies were picked and transferred to new individual 1/2 PDA plates containing appropriate antibiotics. Single-spore isolation of each transformant was performed as for single-deletion mutants, except that the medium contained both antibiotics.

\section{Confirmation of $F$. graminearum mutants.}

Site-directed deletion of native genes in $F$. graminearum mutants was confirmed by PCR. Transformants were grown in $4 \mathrm{ml}$ of liquid CM (Leslie et al. 2006) for 3 to 4 days at $25^{\circ} \mathrm{C}$, under constant shaking at $150 \mathrm{rpm}$. Afterward liquid CM cultures were centrifuged at $2,537 \times g$ for $10 \mathrm{~min}$ to concentrate mycelium, the supernatant was poured off, and the cultures were washed with $8 \mathrm{ml}$ of sterilized deionized $\mathrm{H}_{2} \mathrm{O}$. Cultures were centrifuged again, and excess water was poured off. Mycelium was transferred to a 2-ml centrifuge tube and was frozen for more than an hour in a $-80^{\circ} \mathrm{C}$ freezer. Frozen samples were then placed on a FreeZone 4.5-liter lyophilizer (LaLabconco Corporation, Kansas City, MO, U.S.A.) overnight, to completely dry, and were then transferred to a fresh $2-\mathrm{ml}$ centrifuge tube. Freeze-dried samples were pulverized, by adding a half volume of $0.7-\mathrm{mm}$ silica beads to each tube of mycelium, and were homogenizeded in a Mini-Bead-Beater-8 (BioSpec Products, Bartlesville, OK, U.S.A.) for 15 s. Tubes were then centrifuged at $16,000 \times g$ for $30 \mathrm{~s}$ to concentrate the pulverized mycelium. DNA was extracted from each tube using the CTAB extraction technique (Gale et al. 2011).
Extracted DNA samples were incubated for a minimum of 2 days to ensure the DNA was fully dissolved into the TrisEDTA buffer.

Site-directed gene deletion and replacement with an antibiotic-resistance selectable marker was confirmed by either an either/or amplification technique or an amplicon size determination technique. In the first case, a forward primer that annealed upstream of the targeted locus was paired with either a reverse primer that annealed to the end of the native gene or a reverse primer that annealed to the end of the antibioticresistance gene. This amplification pair would indicate whether the full native gene remained in its resident locus or if the full antibiotic-resistance gene was inserted into the locus. In the second case, the primer pair flank the $5^{\prime}$ and $3^{\prime}$ of the manipulated locus and the size of the resulting amplicon indicates whether the native locus or the resistance gene are present at that locus.

\section{Generation of conidial suspensions of $\boldsymbol{F}$. graminearum.}

Conidial suspensions of $F$. graminearum mutants were made by growing each genotype in $50 \mathrm{ml}$ of carboxy-methyl cellulose (CMC) medium (Cappellini and Peterson 1965) for about 5 days. Conidia were collected by filtering CMC through Miracloth to remove mycelia. CMC filtrate was then centrifuged at $2,537 \times g$ for $10 \mathrm{~min}$ to concentrate conidia, was decanted, was washed with $25 \mathrm{ml}$ of sterile water, was centrifuged and decanted again, and was then brought to $5 \mathrm{ml}$ with sterile water. Concentrated conidial suspensions were enumerated using a hemocytometer and were standardized to $2 \times$ $10^{4}$ conidia per milliliter for in-vitro analyses or $1 \times 10^{6}$ conidia per milliliter for in-planta analyses.

\section{Growth and phenotypic responses of the $\Delta s s o 2$ mutant.}

A 3-mm-diameter plug of $F$. graminearum wild type or $\Delta$ sso2 deletion mutant culture was plated on carrot agar, 1/2 PDA, CD, CM, MM, and V8 agar to assess vegetative growth. Deletion mutants were tested against the fungicides benomyl and tebuconazole, the plant phytoalexin BOA (2-benzoxazolinone), and hydrogen peroxide $\left(\mathrm{H}_{2} \mathrm{O}_{2}\right)$. Solid chemicals were dissolved in DMSO to a stock concentration of 50 to $100 \mathrm{mg} / \mathrm{ml}$ and were added to $1 / 2 \mathrm{PDA}$ or CM for a final working concentration of $0.375 \mu \mathrm{g} / \mathrm{ml}$ (tebuconazole), $1.0 \mu \mathrm{g} / \mathrm{ml}$ (benomyl), or $250 \mu \mathrm{g} / \mathrm{ml}$ (BOA). Final DMSO concentration in media was $0.1 \%$. Hydrogen peroxide was added to $1 / 2$ PDA for a final concentration of $0.03 \%$. Plates were grown in triplicate at $25^{\circ} \mathrm{C}$ for 3 days before determining the area (in square millimeters) of hyphal growth, using a Carestream 4000MM Pro Image Station with Carestream Molecular Imaging Software v.5.2.2.15761 (Carestream Health, Inc., Rochester, NY, U.S.A.). Phenotyping images were taken at 5 days to allow for color maturation.

To determine biomass, a 3-mm-diameter plug of wild-type F. graminearum or $\Delta s s o 2$ mutant mycelium was inoculated in $5 \mathrm{ml}$ of $1 / 2 \mathrm{PDB}$ and was incubated at $25^{\circ} \mathrm{C}$ and $150 \mathrm{rpm}$ for 3 days. After the incubation period, cultures were filtered through Mira-cloth, mycelium was scraped off the filter using a sterile loop and was transferred to a tared 14-ml tube. Filtered mycelium was frozen at $-80^{\circ} \mathrm{C}$ overnight and was lyophilized to dryness. Biomass was determined by weight of the 14-ml tube containing lyophilized mycelium minus the tared weight of the empty tube. To determine $\mathrm{pH}$ of liquid cultures, a 3-mmdiameter plug of wild type or $\Delta s s o 2$ mutant mycelium was inoculated into $5 \mathrm{ml}$ of $1 / 2 \mathrm{PDB}$, with a starting $\mathrm{pH}$ of 5.3 , and was incubated as described above. After the incubation period, the $\mathrm{pH}$ of the cultures was determined using a Beckman Coulter $350 \mathrm{pH}$ meter (Beckman Coulter, Brea, CA, U.S.A.) connected to an InLab Micro $\mathrm{pH}$ electrode (Mettler Toledo, Columbus, 
$\mathrm{OH}$, U.S.A.). Biomass and $\mathrm{pH}$ cultures were conducted in triplicate.

The $F$. graminearum $\Delta$ sso2 mutant was tested against 120 xenobiotics, using the Biolog PM21-PM25 phenotyping microarray system. Additionally, the Biolog SPF2 carbon utilization plate was used to analyze growth on 95 different carbon sources. Conidial suspensions of wild type and $\Delta s s o 2$ mutant genotypes were normalized to $1 \times 10^{6}$ conidia per milliliter in proprietary Biolog inoculation fluid and $100 \mu \mathrm{l}$ of this suspension was inoculated into each of the 96 wells of the Biolog phenotyping microarray plates. For the SPF2 plate, conidial suspensions of $F$. graminearum were made in a $0.2 \%$ carrageenan solution. Plates were sealed with parafilm and were incubated in the dark at $25^{\circ} \mathrm{C}$ for 3 to 4 days. Optical density $(600 \mathrm{~nm})$ measurements were taken at the end of the incubation period using a Synergy H1 Reader (BioTek Instruments, Winooski, VT, U.S.A.).

To determine the number of bulbous cells produced by the $\Delta$ sso2 mutant, spherical bulbous cells that were wider than $10 \mu \mathrm{m}$ in diameter were counted from $\mathrm{z}$ stacks of TBI culture grown for $48 \mathrm{~h}$. Liquid cultures were imaged by bright field microscopy, using a 10x objective on a Nikon Eclipse 90i microscope. The number of spherical cells were counted in three $\mathrm{z}$ stacks of three biological replicates of each strain (wild type [PH1], Tri12-GFP, $\Delta$ sso2/Tri12-GFP). The mean number of spherical cells and the standard deviation were determined from nine measurements per strain.

\section{Localization of an RFP-tagged Sso2.}

Sso2 was N-terminal tagged with TagRFP-T (RFP), using a fusion PCR-based method (Szewczyk et al. 2006). RFP was amplified from plasmid pAL12-Lifeact (Lichius and Read 2010). Regions flanking the Sso2 start codon were amplified from wild-type $F$. graminearum genomic DNA. Primers were modified from previously published methods (Hong et al. 2010), utilizing RFP tails rather than GFP tails. Wild-type protoplasts were cotransformed, using the fusion PCR product and the neomycin-resistance gene cassette amplified from plasmid pSM334 (Fuchs et al. 2004). Transformations were conducted as described above. Selection for neomycin resistance used $150 \mu \mathrm{g}$ of geneticin per milliliter on the bottom layer and $250 \mu \mathrm{g}$ per milliliter on the top layer of the transformation plates. Transformants were picked onto V8 medium containing $250 \mu \mathrm{g}$ of geneticin per milliliter and single spores were isolated as described above. Transformant DNA was extracted using a Qiagen DNeasy plant mini kit (Qiagen, Valencia, CA, U.S.A.). RFP tagging was verified using a primer inside RFP and a primer outside the flanking region, primers for the $S s o 2$ coding region, primers for RFP, and primers for the neomycin cassette (Supplementary Table S1).

To image the Sso2-RFP strain, $100 \mu \mathrm{l}$ of a $0.5 \times 10^{6}$ conidia/ml solution was inoculated into $5 \mathrm{ml}$ of PDB and was incubated for $30 \mathrm{~h}$ at $25^{\circ} \mathrm{C}$ and $150 \mathrm{rpm}$. Afterward, the mycelium was concentrated by pipetting the culture up and down in a 1,000 $\mu$ l-pipette with the tip cut to form a larger opening. Concentrated mycelium was placed on a microscope slide, was covered with a cover slip, and was sealed with clear nail polish. Mycelium was imaged on a Nikon 90i microscope connected to a Nikon DS-Ri1 camera, using Nikon NIS Elements AR software. The RFP fluorescence was visualized with a Texas Red filter with an excitation of 540 to $580 \mathrm{~nm}$ and emissions of 593 to $668 \mathrm{~nm}$.

In-vitro DON accumulation of $\boldsymbol{F}$. graminearum mutants.

The DON accumulation of wild-type $F$. graminearum and $\Delta s s o 2$ and $\Delta a b c 1$ mutants was analyzed in both toxin non- inducing- (NIM) and toxin induction (TBI) liquid media (Menke et al. 2012). TBI contains putrescine as the sole nitrogen source, which has been shown previously to induce DON production (Gardiner et al. 2009a). NIM contains an equimolar concentration of sodium nitrate rather than putrescine as the sole nitrogen source. A $100-\mu l$ volume of $2 \times$ NIM or TBI was added to the wells of a 96-well microtiter plate, with each plate receiving either NIM or TBI (i.e., no cross mixing of media types in a plate). Each genotype of $F$. graminearum was randomly assigned to five wells of the 96-well plate. A $100-\mu$ l inoculation of $2 \times 10^{4}$ conidia per milliliter of each $F$. graminearum genotype was added to the randomly assigned wells. Plates were incubated in the dark for 6 days.

After 6 days of growth, samples were collected for DON accumulation analysis. Using a multichannel pipette, $125 \mu \mathrm{l}$ of media were removed from each well of the 96-well plate and were transferred to labeled PCR strip-tubes and were reorganized by $F$. graminearum genotype. PCR tubes were centrifuged to pellet any mycelium that may have been accidentally transferred and $100 \mu \mathrm{l}$ of supernatant was transferred from PCR tubes to labeled 1-dram screw-cap vials, which were frozen at $-80^{\circ} \mathrm{C}$ for at least $1 \mathrm{~h}$ and were lyophilized overnight to remove any water. Freeze-dried samples were analyzed for DON, 3ADON, and 15-ADON by gas chromatography mass spectrometry (GC-MS), using methods previously described (Goswami and Kistler 2005). Tests were repeated three times, yielding 15 replicates for each genotype.

\section{In-planta FHB symptoms and DON accumulation of $\boldsymbol{F}$. graminearum mutants.}

The ability of wild-type $F$. graminearum and $\Delta s s o 2$ and $\Delta a b c l$ deletion mutants to cause FHB symptoms (bleached, shriveled, necrotic grains) and accumulate DON was evaluated in wheat cultivar Norm, modified from previous methods (Goswami and Kistler 2005; Menke et al. 2012). Plants were grown in soil in an $18^{\circ} \mathrm{C}$ growth chamber under a 16-h light and 8-h dark cycle until anthesis. Upon anthesis, the fifth fully formed spikelet on each head was wounded and inoculated with $10 \mu \mathrm{l}$ of $1 \times 10^{6}$-conidia/ml water with $0.1 \%$ (vol/vol) Triton 60 . Five replicates of each $F$. graminearum genotype were used in addition to a mock inoculation consisting of $10 \mu \mathrm{l}$ of sterile water with $0.1 \%$ ( vol/vol) Triton 60 . Inoculated plants were placed into a dew chamber set to mist plants for $30 \mathrm{~s}$ every 4 min for 2 days, as high humidity has been shown to increase infection success. After 2 days, plants were moved into a $18^{\circ} \mathrm{C}$ growth chamber under a 16-h light and 8-h dark cycle for another 12 days. Tests were repeated five times, yielding 25 replicates for each genotype.

Once the 2-week incubation period was complete, the inoculated heads were scored for FHB disease symptoms by counting the number of diseased spikelets, up to 10 , surrounding the inoculated spikelet. The inoculated spikelet was then removed from the wheat head and was weighed in a tared 1-dram screw-cap vial to obtain its wet mass (Menke et al. 2012). Samples were frozen at $-80^{\circ} \mathrm{C}$ overnight and were analyzed for DON, 3-ADON, and 15-ADON concentrations by GC-MS, using methods previously described (Goswami and Kistler 2005).

\section{Transcriptional impact}

of $\boldsymbol{F}$. graminearum deletion mutants.

$F$. graminearum wild-type strain $\mathrm{PH} 1$ and the $\Delta s s o 2$ and $\Delta a b c 1$ mutants were grown on 1/2 PDA for 5 to 7 days. A fungal plug was transferred from 1/2 PDA into $100 \mathrm{ml}$ of CMC medium in a $250-\mathrm{ml}$ flask and was incubated at $25^{\circ} \mathrm{C}$ and $150 \mathrm{rpm}$ for 
5 days. CMC filtrate was then centrifuged at $2,537 \times g$ for $10 \mathrm{~min}$, to concentrate conidia, which were enumerated using a hemocytometer. Conidia were inoculated into 50-ml TBI medium at a concentration of $1 \times 10^{4}$ conidia per milliliter and were incubated at $28^{\circ} \mathrm{C}$ and $150 \mathrm{rpm}$ for 2 days in total darkness. A total of three replicate TBI cultures were used. After 2 days of growth, mycelia were washed twice with sterile $\mathrm{H}_{2} \mathrm{O}$, were blotted with filter paper to remove excess $\mathrm{H}_{2} \mathrm{O}$, then were flashfrozen in liquid nitrogen and were lyophilized overnight. RNA was isolated from fungal tissue using a modified protocol of the RNeasy plant mini total RNA extraction kit (Qiagen). Modifications include using TRIzol reagent (Life Technologies, Inc., Carlsbad, CA, U.S.A.) and chloroform in place of Buffer RLT or Buffer RLC during cellular lysis steps, foregoing use of QIAShredder columns, extending incubation time after adding reagents, and extending centrifugation times from $15 \mathrm{~s}$ to 30 to $120 \mathrm{~s}$. Sequence data were conducted, via RNA-seq, on an Illumina HiSeq 2500, High Output Mode with 50-bp paired-end reads, at the University of Minnesota Genomics Center. RNAseq data has been submitted to GenBank and is available with the accession number PRJNA580269.

Data quality in fastq files was assessed using FastQC. Lowquality bases and adapter sequences were removed using Trimmomatic (Bolger et al. 2014). Reads are aligned using Hisat2 (Kim et al. 2015). Fragment per kilobase per million reads expression values were generated using Cuffquant and Cufnorm from the Cufflinks package (Trapnell et al. 2012). Raw read counts were generated using featureCounts from the RSubread package (Liao et al. 2019). GO analysis was conducted using the Goseq tool in Galaxy (Young et al. 2010). Enrichment of GO categories was performed with the GO Enrichment tool in FungiDB (Basenko et al. 2018). Differential gene expression analysis was carried out by edgeR package (Robinson et al. 2010).

\section{Data analysis.}

F. graminearum growth rates, bulbous cell formation, FHB disease symptoms, and DON concentrations were analyzed in R statistical software (R Core Team 2017). Data were analyzed by either Student's $t$ test or a one-way analysis of variance (ANOVA) with a Tukey's posthoc test to compare all pair-wise interactions. Student's $t$ test results are shown with asterisks to illustrate significant difference from the control group, and ANOVA results are shown with significance groupings as determined by Tukey's posthoc test. Graphical representations of data were generated in Microsoft Excel.

\section{ACKNOWLEDGMENTS}

The authors acknowledge the Minnesota Supercomputing Institute (MSI) at the University of Minnesota for providing resources that contributed to the research results reported within this paper.

\section{LITERATURE CITED}

Abou Ammar, G., Tryono, R., Döll, K., Karlovsky, P., Deising, H. B., and Wirsel, S. G. R. 2013. Identification of ABC transporter genes of Fusarium graminearum with roles in azole tolerance and/or virulence. PLoS One 8:e79042.

Alexander, N. J., McCormick, S. P., and Hohn, T. M. 1999. TRI12, a trichothecene efflux pump from Fusarium sporotrichioides: Gene isolation and expression in yeast. Mol. Gen. Genet. 261: 977-984.

Ashley, J. N., Hobbs, B. C., and Raistrick, H. 1937. Studies in the biochemistry of micro-organisms: The crystalline colouring matters of Fusarium culmorum (W. G. Smith) Sacc. and related forms. Biochem. J. 31:385-397.

Bahadoor, A., Brauer, E. K., Bosnich, W., Schneiderman, D., Johnston, A., Aubin, Y., Blackwell, B., Melanson, J. E., and Harris, L. J. 2018. Gramillin
A and B: Cyclic lipopeptides identified as the nonribosomal biosynthetic products of Fusarium graminearum. J. Am. Chem. Soc. 140:16783-16791. Basenko, E. Y., Pulman, J. A., Shanmugasundram, A., Harb, O. S., Crouch, K., Starns, D., Warrenfeltz, S., Aurrecoechea, C., Stoeckert, C. J., Jr., Kissinger, J. C., Roos, D. S., and Hertz-Fowler, C. 2018. FungiDB: An integrated bioinformatic resource for fungi and oomycetes. J. Fungi (Basel) 4. Published online.

Boenisch, M. J., Blum, A., Broz, K. L., Gardiner, D. M., and Kistler, H. C. 2019. Nanoscale enrichment of the cytosolic enzyme trichodiene synthase near reorganized endoplasmic reticulum in Fusarium graminearum. Fungal Genet. Biol. 124:73-77.

Boenisch, M. J., Broz, K. L., Purvine, S. O., Chrisler, W. B., Nicora, C. D., Connolly, L. R., Freitag, M., Baker, S. E., and Kistler, H. C. 2017. Structural reorganization of the fungal endoplasmic reticulum upon induction of mycotoxin biosynthesis. Sci. Rep. 7:44296.

Bolger, A. M., Lohse, M., and Usadel, B. 2014. Trimmomatic: A flexible trimmer for Illumina sequence data. Bioinformatics 30:2114-2120.

Cappellini, R. A., and Peterson, J. L. 1965. Macroconidium formation in submerged cultures by a non-sporulating strain of Gibberella zeae. Mycologia 57:962.

Chen, Y., Kistler, H. C., and Ma, Z. 2019. Fusarium graminearum trichothecene mycotoxins: Biosynthesis, regulation, and management. Annu. Rev. Phytopathol. 57:15-39.

Cheng, W., Song, X. S., Li, H. P., Cao, L. H., Sun, K., Qiu, X. L., Xu, Y. B., Yang, P., Huang, T., Zhang, J. B., Qu, B., and Liao, Y. C. 2015. Hostinduced gene silencing of an essential chitin synthase gene confers durable resistance to Fusarium head blight and seedling blight in wheat Plant Biotechnol. J. 13:1335-1345.

Desmond, O. J., Manners, J. M., Stephens, A. E., Maclean, D. J., Schenk, P. M., Gardiner, D. M., Munn, A. L., and Kazan, K. 2008. The Fusarium mycotoxin deoxynivalenol elicits hydrogen peroxide production, programmed cell death and defence responses in wheat. Mol. Plant Pathol. 9:435-445.

Flynn, C. M., Broz, K., Jonkers, W., Schmidt-Dannert, C., and Kistler, H. C. 2019. Expression of the Fusarium graminearum terpenome and involvement of the endoplasmic reticulum-derived toxisome. Fungal Genet. Biol. 124:78-87.

Frandsen, R. J. N., Schütt, C., Lund, B. W., Staerk, D., Nielsen, J., Olsson, S., and Giese, H. 2011. Two novel classes of enzymes are required for the biosynthesis of aurofusarin in Fusarium graminearum. J. Biol. Chem. 286:10419-10428.

Fried, H. M., and Warner, J. R. 1981. Cloning of yeast gene for trichodermin resistance and ribosomal protein L3. Proc. Natl. Acad. Sci. U.S.A. 78:238-242.

Fuchs, U., Czymmek, K. J., and Sweigard, J. A. 2004. Five hydrophobin genes in Fusarium verticillioides include two required for microconidial chain formation. Fungal Genet. Biol. 41:852-864.

Gale, L. R., Harrison, S. A., Ward, T. J., O’Donnell, K., Milus, E. A., Gale, S. W., and Kistler, H. C. 2011. Nivalenol-type populations of Fusarium graminearum and $F$. asiaticum are prevalent on wheat in southern Louisiana. Phytopathology 101:124-134.

Gardiner, D. M., Kazan, K., and Manners, J. M. 2009a. Nutrient profiling reveals potent inducers of trichothecene biosynthesis in Fusarium graminearum. Fungal Genet. Biol. 46:604-613.

Gardiner, D. M., Osborne, S., Kazan, K., and Manners, J. M. 2009b. Low $\mathrm{pH}$ regulates the production of deoxynivalenol by Fusarium graminearum. Microbiology 155:3149-3156.

Gardiner, D. M., Stephens, A. E., Munn, A. L., and Manners, J. M. 2013. An ABC pleiotropic drug resistance transporter of Fusarium graminearum with a role in crown and root diseases of wheat. FEMS Microbiol. Lett. 348:36-45.

Garreau de Loubresse, N., Prokhorova, I., Holtkamp, W., Rodnina, M. V., Yusupova, G., and Yusupov, M. 2014. Structural basis for the inhibition of the eukaryotic ribosome. Nature 513:517-522.

Giraldo, M. C., Dagdas, Y. F., Gupta, Y. K., Mentlak, T. A., Yi, M., Martinez-Rocha, A. L., Saitoh, H., Terauchi, R., Talbot, N. J., and Valent, B. 2013. Two distinct secretion systems facilitate tissue invasion by the rice blast fungus Magnaporthe oryzae. Nat. Commun. 4:1996.

Giraldo, M. C., and Valent, B. 2013. Filamentous plant pathogen effectors in action. Nat. Rev. Microbiol. 11:800-814.

Goswami, R. S. 2012. Targeted gene replacement in fungi using a splitmarker approach. Methods Mol. Biol. 835:255-269.

Goswami, R. S., and Kistler, H. C. 2005. Pathogenicity and in planta mycotoxin accumulation among members of the Fusarium graminearum species complex on wheat and rice. Phytopathology 95:1397-1404.

Gupta, G. D., and Heath, I. B. 2002. Predicting the distribution, conservation, and functions of SNAREs and related proteins in fungi Fungal Genet. Biol. 36:1-21. 
Hong, S. Y., So, J., Lee, J., Min, K., Son, H., Park, C., Yun, S. H., and Lee, Y. W. 2010. Functional analyses of two syntaxin-like SNARE genes, $G z S Y N 1$ and GzSYN2, in the ascomycete Gibberella zeae. Fungal Genet. Biol. 47:364-372

Hutagalung, A. H., and Novick, P. J. 2011. Role of Rab GTPases in membrane traffic and cell physiology. Physiol. Rev. 91:119-149.

Imazaki, I., and Kadota, I. 2015. Molecular phylogeny and diversity of Fusarium endophytes isolated from tomato stems. FEMS Microbiol. Ecol. 91:fiv098.

Jonkers, W., Dong, Y., Broz, K., and Kistler, H. C. 2012. The Wor1-like protein Fgp1 regulates pathogenicity, toxin synthesis and reproduction in the phytopathogenic fungus Fusarium graminearum. PLoS Pathog. 8: e1002724.

Kienle, N., Kloepper, T. H., and Fasshauer, D. 2009. Phylogeny of the SNARE vesicle fusion machinery yields insights into the conservation of the secretory pathway in fungi. BMC Evol. Biol. 9:19.

Kim, D., Langmead, B., and Salzberg, S. L. 2015. HISAT: A fast spliced aligner with low memory requirements. Nat. Methods 12:357-360.

Kim, J. E., Kim, J. C., Jin, J.-M., Yun, S. H., and Lee, Y. W. 2008. Functional characterization of genes located at the aurofusarin biosynthesis gene cluster in Gibberella zeae. Plant Pathol. J. 24:8-16.

Koch, A., Biedenkopf, D., Furch, A., Weber, L., Rossbach, O., Abdellatef, E., Linicus, L., Johannsmeier, J., Jelonek, L., Goesmann, A., Cardoza, V., McMillan, J., Mentzel, T., and Kogel, K. H. 2016. An RNAi-based control of Fusarium graminearum infections through spraying of long dsRNAs involves a plant passage and is controlled by the fungal silencing machinery. PLoS Pathog. 12:e1005901.

Kovalchuk, A., and Driessen, A. J. 2010. Phylogenetic analysis of fungal ABC transporters. BMC Genomics 11:177.

Kuratsu, M., Taura, A., Shoji, J. Y., Kikuchi, S., Arioka, M., and Kitamoto, K. 2007. Systematic analysis of SNARE localization in the filamentous fungus Aspergillus oryzae. Fungal Genet. Biol. 44:1310-1323.

Lee, S., Son, H., Lee, J., Lee, Y. R., and Lee, Y. W. 2011. A putative ABC transporter gene, $Z R A 1$, is required for zearalenone production in Gibberella zeae. Curr. Genet. 57:343-351.

Leslie, J. F., Summerell, B. A., and Bullock, S. 2006. The Fusarium Laboratory Manual. Wiley, New York.

Li, B., Dong, X., Zhao, R., Kou, R., Zheng, X., and Zhang, H. 2019. The tSNARE protein FgPep12, associated with FgVam7, is essential for ascospore discharge and plant infection by trafficking $\mathrm{Ca}^{2+}$ ATPase fgneol between golgi and endosome/ vacuole in Fusarium graminearum. PLoS Pathog. 15:e1007754.

Li, B., Liu, L., Li, Y., Dong, X., Zhang, H., Chen, H., Zheng, X., and Zhang, Z. 2017. The FgVps39-FgVam7-FgSso1 complex mediates vesicle trafficking and is important for the development and virulence of Fusarium graminearum. Mol. Plant-Microbe Interact. 30:410-422.

Liao, Y., Smyth, G. K., and Shi, W. 2019. The R package Rsubread is easier, faster, cheaper and better for alignment and quantification of RNA sequencing reads. Nucleic Acids Res. 47:e47.

Lichius, A., and Read, N. D. 2010. A versatile set of Lifeact-RFP expression plasmids for live-cell imaging of F-actin in filamentous fungi. Fungal Genet. Rep. 57:8-14.

Lofgren, L. A., LeBlanc, N. R., Certano, A. K., Nachtigall, J., LaBine, K. M., Riddle, J., Broz, K., Dong, Y., Bethan, B., Kafer, C. W., and Kistler, H. C. 2018. Fusarium graminearum: Pathogen or endophyte of North American grasses? New Phytol. 217:1203-1212.

Lupton, S. D., Brunton, L. L., Kalberg, V. A., and Overell, R. W. 1991. Dominant positive and negative selection using a hygromycin phosphotransferase-thymidine kinase fusion gene. Mol. Cell. Biol. 11: 3374-3378.

Lysøe, E., Seong, K. Y., and Kistler, H. C. 2011. The transcriptome of Fusarium graminearum during the infection of wheat. Mol. PlantMicrobe Interact. 24:995-1000.

Maier, F. J., Miedaner, T., Hadeler, B., Felk, A., Salomon, S., Lemmens, M., Kassner, H., and Schäfer, W. 2006. Involvement of trichothecenes in fusarioses of wheat, barley and maize evaluated by gene disruption of the trichodiene synthase (Tri5) gene in three field isolates of different chemotype and virulence. Mol. Plant Pathol. 7:449-461.

McCormick, S. P., Alexander, N. J., and Harris, L. J. 2010. CLM1 of Fusarium graminearum encodes a longiborneol synthase required for culmorin production. Appl. Environ. Microbiol. 76:136-141.
Medentsev, A. G., Arinbasarova, A. Iu., and Akimenko, V. K. 2005. [Biosynthesis of naphthoquinone pigments by fungi of the genus Fusarium]. Prikl. Biokhim. Mikrobiol. 41:573-577.

Menke, J., Dong, Y., and Kistler, H. C. 2012. Fusarium graminearum Tri12p influences virulence to wheat and trichothecene accumulation. Mol. Plant-Microbe Interact. 25:1408-1418.

Menke, J., Weber, J., Broz, K., and Kistler, H. C. 2013. Cellular development associated with induced mycotoxin synthesis in the filamentous fungus Fusarium graminearum. PLoS One 8:e63077.

Pasquali, M., and Kistler, C. 2006. Gibberella zeae ascospore production and collection for microarray experiments. J. Vis. Exp. 1:115.

Pestka, J. J. 2007. Deoxynivalenol: Toxicity, mechanisms and animal health risks. Anim. Feed Sci. Technol. 137:283-298.

Proctor, R. H., Hohn, T. M., and McCormick, S. P. 1995. Reduced virulence of Gibberella zeae caused by disruption of a trichothecene toxin biosynthetic gene. Mol. Plant-Microbe Interact. 8:593-601.

Proctor, R. H., McCormick, S. P., Alexander, N. J., and Desjardins, A. E. 2009. Evidence that a secondary metabolic biosynthetic gene cluster has grown by gene relocation during evolution of the filamentous fungus Fusarium. Mol. Microbiol. 74:1128-1142.

Qi, T., Guo, J., Peng, H., Liu, P., Kang, Z., and Guo, J. 2019. Host-induced gene silencing: A powerful strategy to control diseases of wheat and barley. Int. J. Mol. Sci. 20:206.

R Core Team. 2017. R: A language and environment for statistical computing.

Read, N. D. 2011. Exocytosis and growth do not occur only at hyphal tips. Mol. Microbiol. 81:4-7.

Robinson, M. D., McCarthy, D. J., and Smyth, G. K. 2010. edgeR: A Bioconductor package for differential expression analysis of digital gene expression data. Bioinformatics 26:139-140.

Rocha, O., Ansari, K., and Doohan, F. M. 2005. Effects of trichothecene mycotoxins on eukaryotic cells: A review. Food Addit. Contam. 22: 369-378.

Szewczyk, E., Nayak, T., Oakley, C. E., Edgerton, H., Xiong, Y., TaheriTalesh, N., Osmani, S. A., and Oakley, B. R. 2006. Fusion PCR and gene targeting in Aspergillus nidulans. Nat. Protoc. 1:3111-3120.

Trapnell, C., Roberts, A., Goff, L., Pertea, G., Kim, D., Kelley, D. R., Pimentel, H., Salzberg, S. L., Rinn, J. L., and Pachter, L. 2012. Differential gene and transcript expression analysis of RNA-seq experiments with TopHat and Cufflinks. Nat. Protoc. 7:562-578.

Valkonen, M., Kalkman, E. R., Saloheimo, M., Penttilä, M., Read, N. D., and Duncan, R. R. 2007. Spatially segregated SNARE protein interactions in living fungal cells. J. Biol. Chem. 282:22775-22785.

Villagrasa, M., Guillamón, M., Labandeira, A., Taberner, A., Eljarrat, E., and Barceló, D. 2006. Benzoxazinoid allelochemicals in wheat: Distribution among foliage, roots, and seeds. J. Agric. Food Chem. 54: 1009-1015.

Wachowska, U., Packa, D., and Wiwart, M. 2017. Microbial inhibition of Fusarium pathogens and biological modification of trichothecenes in cereal grains. Toxins (Basel) 9:408.

Wang, Q., Jiang, C., Wang, C., Chen, C., Xu, J. R., and Liu, H. 2017. Characterization of the two-speed subgenomes of Fusarium graminearum reveals the fast-speed subgenome specialized for adaption and infection. Front. Plant Sci. 8:140.

Waweru, B., Turoop, L., Kahangi, E., Coyne, D., and Dubois, T. 2014. Nonpathogenic Fusarium oxysporum endophytes provide field control of nematodes, improving yield of banana (Musa sp.). Biol. Control 74: 82-88.

Wilson, W., Dahl, B., and Nganje, W. 2018. Economic costs of Fusarium head blight, scab and deoxynivalenol. World Mycotoxin J. 11:291-302.

Young, M. D., Wakefield, M. J., Smyth, G. K., and Oshlack, A. 2010. Gene ontology analysis for RNA-seq: Accounting for selection bias. Genome Biol. 11:R14.

Zhang, H., Li, B., Fang, Q., Li, Y., Zheng, X., and Zhang, Z. 2016. SNARE protein FgVam7 controls growth, asexual and sexual development, and plant infection in Fusarium graminearum. Mol. Plant Pathol. 17: 108-119.

Zheng, H., Zheng, W., Wu, C., Yang, J., Xi, Y., Xie, Q., Zhao, X., Deng, X., Lu, G., Li, G., Ebbole, D., Zhou, J., and Wang, Z. 2015. Rab GTPases are essential for membrane trafficking-dependent growth and pathogenicity in Fusarium graminearum. Environ. Microbiol. 17: 4580-4599. 